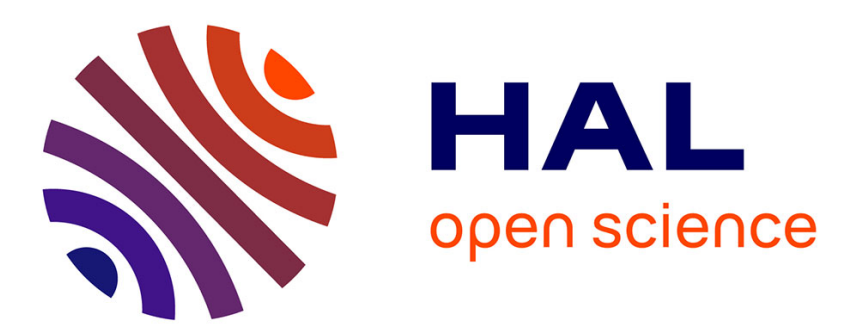

\title{
A multiple criteria sorting method where each category is characterized by several reference actions: The Electre Tri-nC method
}

\author{
Juscelino Almeida Dias, José Figueira, Bernard Roy
}

\section{- To cite this version: \\ Juscelino Almeida Dias, José Figueira, Bernard Roy. A multiple criteria sorting method where each category is characterized by several reference actions: The Electre Tri-nC method. 2010. hal- 00877016}

\section{HAL Id: hal-00877016 https://hal.science/hal-00877016}

Preprint submitted on 25 Oct 2013

HAL is a multi-disciplinary open access archive for the deposit and dissemination of scientific research documents, whether they are published or not. The documents may come from teaching and research institutions in France or abroad, or from public or private research centers.
L'archive ouverte pluridisciplinaire HAL, est destinée au dépôt et à la diffusion de documents scientifiques de niveau recherche, publiés ou non, émanant des établissements d'enseignement et de recherche français ou étrangers, des laboratoires publics ou privés. 


\title{
A multiple criteria sorting method where each category is characterized by several reference actions: The ELECTRE TRI-NC method
}

\author{
J. Almeida-Dias ${ }^{\mathrm{a}, \mathrm{b}, *}$, J. R. FigueirA ${ }^{\mathrm{c}, * *}$, B. Roy ${ }^{\mathrm{b}}$ \\ ${ }^{a}$ CEG-IST, Instituto Superior Técnico, Universidade Técnica de Lisboa, Lisbon, Portugal \\ ${ }^{b}$ LAMSADE, Université Paris-Dauphine, Paris, France \\ ${ }^{c}$ INPL, École des Mines de Nancy, Laboratoire LORIA, Nancy, France
}

\begin{abstract}
This paper presents ELECTRE TRI-NC, a new sorting method which takes into account several reference actions for characterizing each category. This new method gives a particular freedom to the decision maker in the co-construction decision aiding process with the analyst to characterize the set of categories, while there is no constraint for introducing only one reference action as typical of each category like in Electre TRI-C (Almeida-Dias et al., 2010). As in such a sorting method, this new sorting method is composed of two joint rules. ELECTRE TRI-NC also fulfills a certain number of natural requirements. Additional results on the behavior of the new method are also provided in this paper, namely the ones with respect to the addition or removal of the reference actions used for characterizing a certain category. A numerical example illustrates the manner in which Electre TRI-NC can be used by a decision maker. A comparison with some related sorting procedures is presented and it allows to conclude that the new method is appropriate to deal with sorting problems.
\end{abstract}

Keywords: Multiple criteria decision aiding, Constructive approach, Sorting, ElEctre TRI-NC, Decision support

\section{Résumé}

\section{Une méthode multicritère de tri où chaque catégorie est caractérisée par plusieurs actions de référence :} la méthode ELECTRE TRI-NC

Cet article présente Electre TRI-NC, une nouvelle méthode de tri qui prend appui sur plusieurs actions de référence pour caractériser chaque catégorie. Cette nouvelle méthode laisse une grande liberté au décideur dans le processus co-constructif d'interaction avec l'analyste pour caractériser l'ensemble des catégories et elle ne le contraint plus à n'introduire qu'une seule action de référence qui soit typique de chaque catégorie comme c'est le cas avec Electre TRIC (Almeida-Dias et al., 2010). Comme dans cette méthode, la nouvelle méthode est constituée de deux règles couplées. Electre TRI-NC vérifie aussi un certain nombre d'exigences naturelles. Des résultats additionnels par rapport au fonctionnement de la nouvelle méthode sont aussi fournis dans cet article, notamment ceux qui concernent l'ajout ou le retrait d'actions de référence utilisées pour caractériser une catégorie donnée. Un exemple numérique montre la façon dont ELECTRE TRI-NC peut être utilisée par un décideur. Une comparaison avec quelques méthodes de tri qui prennent appui sur des actions de référence comme point de départ est mise en évidence pour permettre de conclure que la nouvelle méthode est appropriée pour traiter les problèmes de tri.

Mots-clés : Aide multicritère à la décision, Approche constructiviste, Problèmes de tri, Electre TRI-NC

\footnotetext{
${ }^{*}$ Corresponding author at: LAMSADE, Université Paris-Dauphine, Place du Maréchal De Lattre de Tassigny, F-75 775 Paris Cedex 16, France, Phone: +331440542 87; Fax: +33144054091.

** Associate member at CEG-IST, Lisbon, Portugal.

Email addresses: judiascv@gmail.com (J. AlmeIdA-Dias), Jose.Figueira@mines.inpl-nancy.fr (J. R. FIGUEIRA), roy@lamsade.dauphine.fr (B. Roy)
} 


\section{Contents}

1 Introduction 2

2 Problem statement 3

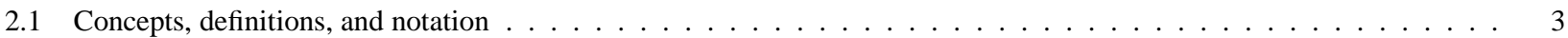

2.2 Structural requirements $\ldots \ldots \ldots \ldots$

3 The Electre Tri-NC method 6

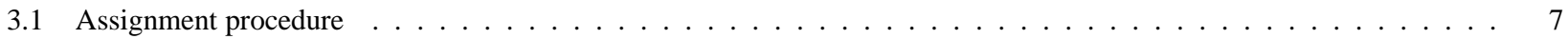

3.2 Foundations of Electre $\mathrm{TRI-NC} \ldots \ldots \ldots \ldots \ldots$

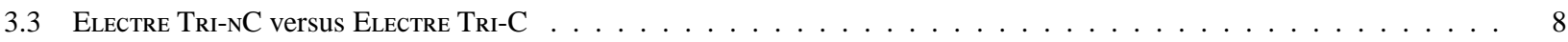

4 Properties of Electre TRI-NC 9

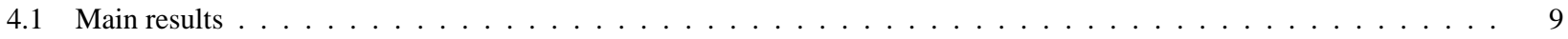

4.2 Adding or removing a characteristic reference action $\ldots \ldots \ldots \ldots$

5 A numerical example 11

6 Comparison with related sorting methods 15

7 Conclusions 16

$\begin{array}{lll}\text { Appendix A } & \text { Proof of Theorem 1 } & 17\end{array}$

Appendix B Proof of Proposition 1 17

Appendix C Proof of Proposition 2 17

Appendix D $\quad$ Proof of Theorem $3 \quad 18$

\section{Introduction}

Sorting problems arise in several real-life activities. In this paper, we are interested in decision aiding contexts in which the objects of a decision (actions, alternatives, ...) must be sorted, or assigned to a set of categories. Such an assignment is based on the evaluation of each action according to multiple criteria. As we have shown in ELECTRE TRI-C framework (Almeida-Dias et al., 2010), there are different manners to deal with the sorting approach. In what follows, we shall present a new approach, in which three assumptions are also taken into account, where only the Assumption 3 differs from the ones in ELECTRE TRI-C.

Assumption 1. The set of categories to which the actions must be assigned to is completely ordered (from the worst to the best, from the lowest priority to the highest priority, from the most risky to the least risky, from the least consensual to the most consensual, and so on). In general, numbering the categories from 1 to q must be coherent with respect to the increasing preferences on the criteria.

Assumption 2. Each category is conceived a priori to receive actions, which will be or might be processed in the same way (in the step that follows the assignment).

Assumption 3. Each category is characterized by a subset of reference actions judged by the decision maker as representative, or informative of the actions that should be assigned to such a category. The decision maker is able, through a co-construction interactive process with the analyst, to provide the performances of the reference actions for characterizing each category according to Assumption 2.

The new method proposed in this paper, called ElECTRE TRI-NC, takes appropriately into account the Assumption 3. As in Electre TRI-C, the objective of Electre TRI-NC is not to discover a pre-existing set of categories where the studied actions would naturally be assigned to. The objective is rather to help decision makers to characterize an appropriate 
set of categories to receive actions according to Assumption 2. We would like to call the attention of the reader to the following aspects. In several concrete decision aiding situations (see, for instance, the examples in Almeida-Dias et al. (2010, Section 1)), it is not possible to refer to an objective set of reference actions defined $a$ priori, which can be used as a starting point for characterizing the set of categories according to Assumption 2. The reference actions should express a willing of the decision maker for characterizing the categories.

The characterization of the categories based on representative reference actions gives a "fuzzy" position with respect to the frontier between a given category and the two consecutive adjacent ones. Some of such characteristic reference actions can be incomparable or even indifferent to some actions to be assigned to the categories. These two reasons seem enough to justify that a given action can be assigned to more than one consecutive categories. Thus, ELECTRE TRI-NC has been conceived to be able to propose to the decision maker all the possible assignments according to Assumption 2.

The rest of this paper is organized as follows. Section 2 is devoted to the problem statement framework. Section 3 presents the assignment procedure, the foundations of ELECTRE TRI-NC, by putting into light new possibilities of such a sorting method. Section 4 introduces the properties of ElECTRE TRI-NC as well as the impact of a new characterization of the set of categories. Section 5 provides a numerical example. Section 6 includes a comparison to other sorting methods, where characteristic reference actions are initially used for representing the willing of the decision maker about the assignment to each category. Finally, the last section offers our concluding remarks and some avenues for future research.

\section{Problem statement}

This section is devoted to the main concepts, definitions, and notation as well as the structural requirements concerning the Electre Tri-NC method.

\subsection{Concepts, definitions, and notation}

Let $A=\left\{a_{1}, a_{2}, \ldots, a_{i}, \ldots\right\}$ denote the set of potential actions. This set of actions can be completely known a priori or it may appear progressively during the decision aiding process. The objective is to assign these actions to a set of completely ordered categories, denoted $C=\left\{C_{1}, C_{2}, \ldots, C_{h}, \ldots, C_{q}\right\}$, with $q \geqslant 2$ (with $q=1$, there is no sorting problem). Suppose that a coherent set of $n$ criteria, denoted $F=\left\{g_{1}, g_{2}, \ldots, g_{j}, \ldots, g_{n}\right\}$, with $n \geqslant 3$, has been defined in order to evaluate any action considered to be assigned to a certain category (see Roy, 1996). Let us notice that if $n<3$, then the concept of concordance is not really pertinent. For such a reason, when using ELECTRE family of methods, it is recommended to have at least three criteria.

Each criterion $g_{j}$ will be considered as a pseudo-criterion, which means that two thresholds are associated to $g_{j}$ : an indifference threshold, $q_{j}$, and a preference threshold, $p_{j}$, such that $p_{j} \geqslant q_{j} \geqslant 0$. These thresholds are introduced in order to take into account the imperfect character of the data from the computation of the performances $g_{j}(a)$, for all $a \in A$, as well as the arbitrariness that affects the definition of the criteria. For more details about the definition of such thresholds, see, for instance, Almeida-Dias et al. (2010, Section 2). Let us notice that the case where $p_{j}=q_{j}=0$, for all $g_{j} \in F$, is not excluded, but such a case must be considered as a very particular realistic case. In what follows, assume, without loss of generality, that all criteria $g_{j} \in F$ are to be maximized, which means that the preferences increase when the criteria performances increase too.

When using the outranking concept, the main idea is that " $a$ outranks $a^{\prime \prime}$ " according to $g_{j}$, denoted $a S_{j} a$, if " $a$ is at least as good as $a^{\prime \prime}$ " on criterion $g_{j}$. Due to the definition of the indifference thresholds, $q_{j}$, it is quite natural to consider that such an assertion is validated, without ambiguity, when $g_{j}(a)-g_{j}\left(a^{\prime}\right) \geqslant-q_{j}$. But, when $-p_{j} \leqslant g_{j}(a)-g_{j}\left(a^{\prime}\right)<-q_{j}$, the possibility of indifference between $a$ and $a^{\prime}$ cannot be excluded. This indifference is less and less credible when $g_{j}(a)-g_{j}\left(a^{\prime}\right)$ moves closer to $-p_{j}$.

Let $\sigma\left(a, a^{\prime}\right)$ denote the credibility of the comprehensive outranking of $a$ over $a^{\prime}$, which reflects the strength of the statement " $a$ outranks $a^{\prime \prime}$ " (denoted $a S a^{\prime}$ ) when taking all the criteria from $F$ into account. This aggregation issue is based on a single vector of weights, denoted $w_{j}$, such that $w_{j}>0, j=1, \ldots, n$, which is associated to the set of criteria. Additionally, a vector of veto thresholds, denoted $v_{j}$, such that $v_{j} \geqslant p_{j}$ can also be associated to the set of criteria. For more details on the computation of $\sigma\left(a, a^{\prime}\right)$, see, for instance, Almeida-Dias et al. (2010, Section 2).

Let us introduce now the set of reference actions. Let $B_{h}=\left\{b_{h}^{r}, r=1, \ldots, m_{h}\right\}$ denote a subset of reference actions introduced to characterize category $C_{h}$, such that $m_{h} \geqslant 1$ and $h=1, \ldots, q$. Notice that $C_{1}$ is the worst category and $C_{q}$ is the best one, with $q \geqslant 2$. Let $B \cup\left\{B_{0}, B_{q+1}\right\}$ denote the set of $(q+2)$ subsets of reference actions, or the set of all reference actions, such that $B=\left\{B_{1}, B_{2}, \ldots, B_{h}, \ldots, B_{q}\right\}$. The two particular subsets of reference actions, denoted $B_{0}=\left\{b_{0}^{1}\right\}$ and 
$B_{q+1}=\left\{b_{q+1}^{1}\right\}$, contains two reference actions defined as follows: $g_{j}\left(b_{0}^{1}\right)$ is the worst possible performance on criterion $g_{j}$, and $g_{j}\left(b_{q+1}^{1}\right)$ is the best possible performance on the same criterion $g_{j}$, for all $g_{j} \in F$. The worst and the best possible performances must be chosen such that, for any action $a$, one has $g_{j}\left(b_{0}^{1}\right)<g_{j}(a)<g_{j}\left(b_{q+1}^{1}\right)$, for all $g_{j} \in F$. Moreover, for all $g_{j} \in F$, one has $g_{j}\left(b_{1}^{r}\right)-g_{j}\left(b_{0}^{1}\right)>0, r=1, \ldots, m_{1}$, and $g_{j}\left(b_{q+1}^{1}\right)-g_{j}\left(b_{q}^{s}\right)>0, s=1, \ldots, m_{q}$.

The comparison of an action $a$ to the characteristic reference actions $b_{h}^{r}, r=1, \ldots, m_{h}$, provides $m_{h}$ credibility indices of each type, $\sigma\left(a, b_{h}^{r}\right)$ and $\sigma\left(b_{h}^{r}, a\right)$. In order to make a judgment regarding the way in which an action $a$ is placed with respect to the category $C_{h}$, it is suitable to find an aggregation operator that allows to obtain a representative credibility index for each action $a$ with respect to each subset of reference actions, $B_{h}, h=1, \ldots, q$. As for the case of decision aiding sorting methods using a set of unordered categories (see, for instance, Perny, 1998; Henriet, 2000; Belacel, 2000; Léger and Martel, 2002), the max operator is also a natural choice in our framework as follows:

Definition 1 (Categorical credibility indices).

(a) $\sigma\left(\{a\}, B_{h}\right)=\max _{r=1, \ldots, m_{h}}\left\{\sigma\left(a, b_{h}^{r}\right)\right\}$

(b) $\sigma\left(B_{h},\{a\}\right)=\max _{s=1, \ldots, m_{h}}\left\{\sigma\left(b_{h}^{s}, a\right)\right\}$

The credibility indices computed according to Definition 1(a) can be interpreted as the categorical outranking degrees of action $a$ over the subset of reference actions $B_{h}$. Similarly, the credibility indices computed according to Definition 1(b) can be interpreted as the categorical outranked degrees of action $a$ over the subset of reference actions $B_{h}$.

The justification for these two interpretations are as follows. The categorical credibility indices, $\sigma\left(\{a\}, B_{h}\right)$ and $\sigma\left(B_{h},\{a\}\right)$, are used for managing the assignment process (see Section 3.1). Thus,

(1) when defining the outranking credibility of an action $a$ over a subset of reference actions $B_{h}, \sigma\left(\{a\}, B_{h}\right)$, it seems natural to impose $a$ priori that such a credibility degree should verify the following two axioms:

Axiom 1. If $B_{h}=\left\{b_{h}^{1}\right\}$, then, for any action $a, \sigma\left(\{a\}, B_{h}\right)=\sigma\left(a, b_{h}^{1}\right)$.

Axiom 2. If $\left|B_{h}\right| \geqslant 2$ and there exists $b_{h}^{r} \in B_{h}$ such that $\sigma\left(a, b_{h}^{r}\right) \leqslant \sigma\left(\{a\}, B_{h}\right)$, then, for any action $a, \sigma\left(\{a\}, B_{h} \backslash\left\{b_{h}^{r}\right\}\right)=$ $\sigma\left(\{a\}, B_{h}\right)$.

Let $B_{h}=\left\{b_{h}^{1}, \ldots, b_{h}^{m_{h}}\right\}$ and $\sigma\left(a, b_{h}^{1}\right) \geqslant \sigma\left(a, b_{h}^{r}\right), r=2, \ldots, m_{h}$. Let us prove that if $\sigma\left(\{a\}, B_{h}\right)$ verifies Axioms 1 and 2, then $\sigma\left(\{a\}, B_{h}\right)=\sigma\left(a, b_{h}^{1}\right)=\max _{r=1, \ldots, m_{h}}\left\{\sigma\left(a, b_{h}^{r}\right)\right\}$. First, if $m_{h}=1$, then, according to Axiom 1, we have $\sigma\left(\{a\}, B_{h}\right)=\sigma\left(a, b_{h}^{1}\right)$. Second, if $m_{h} \geqslant 2$, then, according to Axiom 2, we have $\sigma\left(\{a\}, B_{h}\right)=\sigma\left(\{a\},\left\{b_{h}^{1}, \ldots, b_{h}^{m_{h}-1}\right\}\right)=$ $\sigma\left(\{a\},\left\{b_{h}^{1}, \ldots, b_{h}^{m_{h}-2}\right\}\right)=\ldots=\sigma\left(a, b_{h}^{1}\right)$.

(2) when defining the outranking credibility of a subset of reference actions $B_{h}$ over an action $a, \sigma\left(B_{h},\{a\}\right)$, it seems natural to impose a priori that such a credibility degree should verify the following two axioms:

Axiom 3. If $B_{h}=\left\{b_{h}^{1}\right\}$, then, for any action $a, \sigma\left(B_{h},\{a\}\right)=\sigma\left(b_{h}^{1}, a\right)$.

Axiom 4. If $\left|B_{h}\right| \geqslant 2$ and there exists $b_{h}^{r} \in B_{h}$ such that $\sigma\left(b_{h}^{r}, a\right) \leqslant \sigma\left(B_{h},\{a\}\right)$, then, for any action $a, \sigma\left(B_{h} \backslash\left\{b_{h}^{r}\right\},\{a\}\right)=$ $\sigma\left(B_{h},\{a\}\right)$.

The proof that leads to the justification of the max operator with respect to Definition 1.b is done in a similar way.

Remark 1. Since for all $b_{h}^{r}, 0 \leqslant \sigma\left(a, b_{h}^{r}\right) \leqslant 1$ and, for all $b_{h}^{s}, 0 \leqslant \sigma\left(b_{h}^{s}, a\right) \leqslant 1$, then $0 \leqslant \sigma\left(\{a\}, B_{h}\right) \leqslant 1$ and $0 \leqslant \sigma\left(B_{h},\{a\}\right) \leqslant 1, h=1, \ldots, q$.

Let $\lambda$ denote a credibility level as the minimum degree of credibility, which is considered or judged necessary by the decision maker for validating or not an outranking statement taking into account all the criteria from $F$. This minimum credibility level takes a value within the range $[0.5,1]$. When comparing an action $a$ to a subset of reference actions $B_{h}$, this credibility level allows to define four $\lambda$-binary relations as follows (see also Proposition 1, in Section 4). 
Definition 2 ( $\lambda$-binary relations).

(a) $\lambda$-outranking: $\{a\} S^{\lambda} B_{h} \Leftrightarrow \sigma\left(\{a\}, B_{h}\right) \geqslant \lambda$.

(b $\left.b_{1}\right) \lambda$-preference: $\{a\} P^{\lambda} B_{h} \Leftrightarrow \sigma\left(\{a\}, B_{h}\right) \geqslant \lambda$ and $\sigma\left(B_{h},\{a\}\right)<\lambda$.

$\left(b_{2}\right) \lambda$-indifference: $\{a\} I^{\lambda} B_{h} \Leftrightarrow \sigma\left(\{a\}, B_{h}\right) \geqslant \lambda$ and $\sigma\left(B_{h},\{a\}\right) \geqslant \lambda$.

(b $\left.b_{3}\right) \lambda$-incomparability: $\{a\} R^{\lambda} B_{h} \Leftrightarrow \sigma\left(\{a\}, B_{h}\right)<\lambda$ and $\sigma\left(B_{h},\{a\}\right)<\lambda$.

In order to conceive the characteristic reference actions, it is required that those belonging to $B_{h+1}$ and the ones belonging to $B_{h}$ define two consecutive distinct categories. This means that it is necessary to impose that each characteristic reference action from $B_{h+1}$ dominates each characteristic reference action from $B_{h}$.

Condition 1 (Dominance). The set of reference actions, $B$, fulfills the dominance condition if and only if $\forall j, g_{j}\left(b_{h+1}^{s}\right)-$ $g_{j}\left(b_{h}^{r}\right) \geqslant 0, s=1, \ldots, m_{h+1} ; r=1, \ldots, m_{h} ; h=1, \ldots,(q-1)$.

Let us notice that if $B_{h}$ has three characteristic reference actions such that $B_{h}=\left\{b_{h}^{r}, b_{h}^{s}, b_{h}^{t}\right\}$, where $b_{h}^{r}$ dominates $b_{h}^{s}$ and $b_{h}^{s}$ dominates $b_{h}^{t}$, then the characteristic reference action $b_{h}^{s}$ can be deleted from $B_{h}$ because such a reference action does not play any role when comparing an action $a$ to the subset $B_{h}$. Therefore, $b_{h}^{s}$ is redundant.

Nevertheless, when considering the possible minimum differences in the performances of the reference actions, the dominance condition is not sufficient for characterizing two consecutive distinct categories. It is necessary to exclude the possibility of having two characteristic reference actions, $b_{h+1}^{s}$ and $b_{h}^{r}$, defined by non significantly different performances on each criterion, since they are not able to characterize distinct categories. Two subsets of reference actions, $B_{h+1}$ and $B_{h}$, characterize two consecutive distinct categories only if at least each reference action belonging to $B_{h+1}$ is weakly preferred to each reference action belonging to $B_{h}$ according to at least one criterion. Therefore, the set of reference actions, $B$, must fulfill the weak separability condition as follows:

Condition 2 (Weak separability). The set of reference actions, B, fulfills the weak separability condition if and only if it fulfills the dominance condition and $\sigma\left(b_{h}^{r}, b_{h+1}^{s}\right)<1, r=1, \ldots, m_{h} ; s=1, \ldots, m_{h+1} ; h=1, \ldots,(q-1)$.

Two additional stronger conditions can also be defined as follows:

Condition 3 (Strict separability). The set of reference actions, B, fulfills the strict separability condition if and only if it fulfills the dominance condition and $\sigma\left(b_{h}^{r}, b_{h+1}^{s}\right)<\frac{1}{2}, r=1, \ldots, m_{h} ; s=1, \ldots, m_{h+1} ; h=1, \ldots,(q-1)$.

Condition 4 (Hyper-strict separability). The set of reference actions, $B$, fulfills the hyper-strict separability condition if and only if it fulfills the dominance condition and $\sigma\left(b_{h}^{r}, b_{h+1}^{s}\right)=0, r=1, \ldots, m_{h} ; s=1, \ldots, m_{h+1} ; h=1, \ldots,(q-1)$.

The above separability conditions are required only between the reference actions that characterize different categories. Let us notice that there is no reason to impose some constraints on the reference actions characterizing the same category, $C_{h}$. This means that when $B_{h}, h=1, \ldots, q$, contains at least two reference actions, introduced to characterize the category $C_{h}, h=1, \ldots, q$, between each pair of such reference actions we can have $\lambda$-indifference, $\lambda$-preference, or $\lambda$-incomparability.

Remark 2. Conditions 1, 2, 3, and 4, which are used within the ElECTRE TRI-NC framework, are based on similar conditions provided by Almeida-Dias et al. (2010).

\subsection{Structural requirements}

The section introduces the structural requirements, which can be viewed as the desirable properties of the ELECTRE TRI-NC method, as follows.

Definition 3 (Structural requirements).

(a) Conformity: each characteristic reference action $b_{h}^{r}, r=1, \ldots, m_{h}$, must be assigned to category $C_{h}, h=1, \ldots, q$.

(b) Homogeneity: two actions must be assigned to the same category when they have the same outranking credibility indices with respect to each one of the characteristic reference actions.

(c) Monotonicity: if an action a strictly dominates $a^{\prime}$, then a is assigned at least to the same category $a^{\prime}$ is assigned to. 
(d) Stability: when applying either a merging or a splitting operation (see Definition 4 below), the actions previously assigned to the non-modified categories will be assigned to the same categories or, possibly, to the new categories, after modification. More precisely:

(1) After merging two consecutive categories:

- any action previously assigned to a non-adjacent category to the modified ones will remain in the same category;

- any action previously assigned to an adjacent category to the modified ones will either be assigned to the same category or to the new category;

- any action previously assigned to a merged category will either be assigned to the new category or to an adjacent category.

(2) After splitting a category into two new consecutive categories:

- any action previously assigned to a non-adjacent category to the modified one will remain in the same category;

- any action previously assigned to an adjacent category to the modified one will either be assigned to the same category or to a new category;

- any action previously assigned to the split category will either be assigned to a new category or to an adjacent category.

Definition 4 (Merging and splitting operations).

(a) Merging operation: two consecutive categories, $C_{h}$ and $C_{h+1}$, will be merged to become a new one, $C_{h}^{\prime}$, characterized by a new subset of reference actions, $B_{h}^{\prime}=\left\{b_{h}^{r^{\prime}}, r^{\prime}=1, \ldots, m_{h}^{\prime}\right\}$, such that, for all $g_{j} \in F$ :

(1) for all $b_{h^{\prime}}^{r^{\prime}}$, there is at least one $b_{h}^{r}$ verifying $g_{j}\left(b_{h}^{r^{\prime}}\right)-g_{j}\left(b_{h}^{r}\right) \geqslant 0$;

(2) for all $b_{h}^{r^{\prime}}$, there is at least one $b_{h+1}^{s}$ verifying $g_{j}\left(b_{h+1}^{s}\right)-g_{j}\left(b_{h}^{r^{\prime}}\right) \geqslant 0$.

(b) Splitting operation: the category $C_{h}$ is split into two new consecutive categories, $C_{h}^{\prime}$ and $C_{h}^{\prime \prime}$, characterized by two new distinct subsets of reference actions, $B_{h}^{\prime}=\left\{b_{h}^{r^{\prime}}, r^{\prime}=1, \ldots, m_{h}^{\prime}\right\}$ and $B_{h}^{\prime \prime}=\left\{b_{h}^{r^{\prime \prime}}, r^{\prime \prime}=1, \ldots, m_{h}^{\prime \prime}\right\}$, respectively, such that:

(1) for all $b_{h+1}^{s}$ and $b_{h}^{r^{\prime \prime}}, \sigma\left(b_{h}^{r^{\prime \prime}}, b_{h+1}^{s}\right)<1$;

(2) for all $b_{h}^{r^{\prime \prime}}$ and $b_{h}^{r^{\prime}}, \sigma\left(b_{h}^{r^{\prime}}, b_{h}^{r^{\prime \prime}}\right)<1$;

(3) for all $b_{h^{\prime \prime}}^{r^{\prime}}$ and $b_{h-1}^{r}, \sigma\left(b_{h-1}^{r}, b_{h}^{r^{\prime}}\right)<1$;

(4) for all $b_{h}^{r^{\prime \prime}}$, there is at least one $b_{h}^{r}$ verifying $g_{j}\left(b_{h}^{r^{\prime \prime}}\right)-g_{j}\left(b_{h}^{r}\right) \geqslant 0$, for all $g_{j} \in F$;

(5) for all $b_{h}^{r^{\prime}}$, there is at least one $b_{h}^{r}$ verifying $g_{j}\left(b_{h}^{r}\right)-g_{j}\left(b_{h}^{r^{\prime}}\right) \geqslant 0$, for all $g_{j} \in F$.

Definition 3(d) implies that the set of reference actions, $B$, will be changed within a co-construction interactive process between the analyst and the decision maker. After a merging operation (Definition 4(a)), the new set of categories becomes $C^{*}=\left\{C_{1}, C_{2}, \ldots, C_{h-1}, C_{h}^{\prime}, C_{h+2}, \ldots, C_{q}\right\}$. This new set of categories is characterized by a new set of reference actions, denoted $B^{*}=\left\{B_{1}, B_{2}, \ldots, B_{h-1}, B_{h}^{\prime}, B_{h+2}, \ldots, B_{q}\right\}$, which trivially fulfills at least the weak separability condition. After a splitting operation (Definition $4(\mathrm{~b})$ ), the new set of categories becomes $C^{*}=\left\{C_{1}, C_{2}, \ldots, C_{h-1}, C_{h}^{\prime}, C_{h}^{\prime \prime}, C_{h+1}, \ldots, C_{q}\right\}$. This new set of categories is characterized by a new set of reference actions, denoted $B^{*}=\left\{B_{1}, B_{2}, \ldots, B_{h-1}, B_{h}^{\prime}, B_{h}^{\prime \prime}\right.$, $\left.B_{h+1}, \ldots, B_{q}\right\}$, which must fulfill at least the weak separability condition.

Let us notice that adding or removing a category are particular cases of a merging or a spitting operation. Additionally, when a category can be characterized by more than one reference action, it is suitable to analyze the impact of a local modification of a category by adding or removing a characteristic reference action to or from each subset of reference actions (see Section 4.2).

\section{The Electre Tri-nC method}

The aim of this section is to present the assignment procedure and the foundations of ELECTRE TRI-NC as well as a briefly comparison to ELECTRE TRI-C. 


\subsection{Assignment procedure}

The Electre Tri-NC assignment procedure is composed of two joint rules, called the descending rule and the ascending rule, which must be used conjointly (and not separately). These rules are based on the same rules proposed for the ElECTRE TRI-C method (Almeida-Dias et al., 2010), while replacing the classical credibility indices by the categorical credibility indices (Definition 1) introduced in Section 2.1. As in EleCTRE TRI-C, both joint rules firstly pre-select a category between two possibles ones, and secondly they select an appropriate category by making use of a selecting function, denoted $\rho\left(\{a\}, B_{h}\right)$, for a possible assignment of each action $a$. Due to the role played by this function, it must fulfill the two following properties:

\section{Property 1.}

(a) $\rho\left(\{a\}, B_{h}\right)$ is a function of $\sigma\left(\{a\}, B_{h}\right)$ and $\sigma\left(B_{h},\{a\}\right)$, where $B_{h}$ is a subset of reference actions, $h=1, \ldots, q$.

(b) Let $C_{h}$ be the pre-selected category for a possible assignment of action a. The chosen condition for selecting $C_{h}$ rather than $C_{h-1}$ or $C_{h+1}$, which is also candidate, must be meaningful. Such a condition is the following one: $\rho\left(\{a\}, B_{h}\right)>\rho\left(\{a\}, B_{s}\right)$, with $s=h-1$ or $s=h+1$ (which depends on one of the two joint rules where the pre-selection is made). Consequently, if an action a has the same performance for each criterion as one of the characteristic reference actions from $B_{h}$, then $\rho\left(\{a\}, B_{h}\right)$ must be strictly greater than $\rho\left(\{a\}, B_{s}\right)$, with $s=h-1$ or $s=h+1$.

Property 2. Let $a$ and $a^{\prime}$ be two actions that allow to pre-select the same category. If a strictly dominates $a^{\prime}$, then $\rho\left(\{a\}, B_{h}\right)>\rho\left(\{a\}, B_{h+1}\right) \Rightarrow \rho\left(\left\{a^{\prime}\right\}, B_{h}\right)>\rho\left(\left\{a^{\prime}\right\}, B_{h+1}\right)$. This implication is equivalent, by the logic negation, to $\rho\left(\left\{a^{\prime}\right\}, B_{h+1}\right) \geqslant$ $\rho\left(\left\{a^{\prime}\right\}, B_{h}\right) \Rightarrow \rho\left(\{a\}, B_{h+1}\right) \geqslant \rho\left(\{a\}, B_{h}\right)$.

Property 1(a) is imposed in order to clarify the arguments in which Electre TRI-NC is founded. Property 1(b) is necessary so that the selected categories by each one of the two joint rules play the appropriate role, which is given by the ELECTRE TRI-NC assignment procedure. Property 2 is necessary in order to fulfill the monotonicity with respect to each one of the two joint rules.

In each one of the joint rules, defined hereafter, we propose to make use of the following selecting function (see also Section 3.2, question (4)):

$$
\rho\left(\{a\}, B_{h}\right)=\min \left\{\sigma\left(\{a\}, B_{h}\right), \sigma\left(B_{h},\{a\}\right)\right\} .
$$

Definition 5 (Descending rule). Choose a credibility level, $\lambda\left(\frac{1}{2} \leqslant \lambda \leqslant 1\right)$. Decrease $h$ from $(q+1)$ until the first value, $t$, such that $\sigma\left(\{a\}, B_{t}\right) \geqslant \lambda\left(C_{t}\right.$ is called the descending pre-selected category):

(a) For $t=q$, select $C_{q}$ as a possible category to assign action $a$.

(b) For $0<t<q$, if $\rho\left(\{a\}, B_{t}\right)>\rho\left(\{a\}, B_{t+1}\right)$, then select $C_{t}$ as a possible category to assign a; otherwise, select $C_{t+1}$.

(c) For $t=0$, select $C_{1}$ as a possible category to assign a.

In the descending rule, a category is pre-selected taking into account that: $B_{t}$ is the highest subset of reference actions such that the statement " $a$ outranks $B_{t}$ " is validated with the chosen credibility level, $\lambda$. In such a case, the possibility of the assignment of action $a$ to the descending pre-selected category $C_{t}$ must be examined. However, taking into account the manner that the subsets of reference actions $B_{t}$ and $B_{t+1}$ were defined, the assignment of action $a$ to $C_{t+1}$ is an alternative that must also be examined (in such a case, the statement " $a$ outranks $B_{t+1}$ " is not validated with the chosen credibility level, $\lambda$ ) because $B_{t+1}$ was not defined to play the role of a subset of upper bounds for the category $C_{t}$.

Definition 6 (Ascending rule). Choose a credibility level, $\lambda\left(\frac{1}{2} \leqslant \lambda \leqslant 1\right)$. Increase $h$ from zero until the first value, $k$, such that $\sigma\left(B_{k},\{a\}\right) \geqslant \lambda\left(C_{k}\right.$ is called the ascending pre-selected category):

(a) For $k=1$, select $C_{1}$ as a possible category to assign action $a$.

(b) For $1<k<(q+1)$, if $\rho\left(\{a\}, B_{k}\right)>\rho\left(\{a\}, B_{k-1}\right)$ then select $C_{k}$ as a possible category to assign a; otherwise, select $C_{k-1}$.

(c) For $k=(q+1)$, select $C_{q}$ as a possible category to assign a. 
In the ascending rule, a category is pre-selected taking into account that: $B_{k}$ is the lowest subset of reference actions such that the statement " $B_{k}$ outranks $a$ " is validated with the chosen credibility level, $\lambda$. In such a case, the possibility of the assignment of action $a$ to the ascending pre-selected category $C_{k}$ must be examined. However, taking into account the manner that the subsets of reference actions $B_{k}$ and $B_{k-1}$ were defined, the assignment of action $a$ to $C_{k-1}$ is an alternative that must also be examined (in such a case, the statement " $B_{k-1}$ outranks $a$ " is not validated with the chosen credibility level, $\lambda$ ) because $B_{k-1}$ was not defined to play the role of a subset of lower bounds for the category $C_{k}$.

Remark 3. If each one of the subsets of reference actions, $B_{h}, h=1, \ldots, q$, has only one characteristic reference action such that $B_{h}=\left\{b_{h}\right\}, h=1, \ldots, q$, then the descending rule (respectively the ascending rule) of ELECTRE TRI-NC does not differ from the descending rule (respectively the ascending rule) of ELECTRE TRI-C (Almeida-Dias et al., 2010).

Electre Tri-nC assignment procedure leads to select a lowest and a highest possible categories to which an action $a$ can be assigned to by using the descending rule and the ascending rule conjointly (and not separately). Therefore, ELECTRE TRI-NC provides as a possible assignment of action $a$ (see Theorem 2, in Section 4.1):

- one category, when the two selected categories are the same;

- two categories, when the two selected categories are consecutive;

- a range of more than two consecutive categories, delimited by the two selected categories.

In what follows, $\Gamma(a)$ denote the range of consecutive categories provided by ELECTRE TRI-NC as possible categories to which an action $a$ can be assigned to.

\subsection{Foundations of Electre Tri-NC}

This section provides the foundations for ELECTRE TRI-NC. They are based on the answers of the following four key questions:

(1) Why to found an assignment procedure on the basis of the categorical credibility indices of types $\sigma\left(\{a\}, B_{h}\right)$ and $\sigma\left(B_{h},\{a\}\right) ?$

(2) Why two joint assignment rules for giving a possible range of consecutive categories in which an action a can be assigned to?

(3) Why to use both descending rule and ascending rule conjointly?

(4) What kind of selecting function, $\rho\left(\{a\}, B_{h}\right)$, guarantees that $B_{h}$ plays the required role according to its meaning?

The answers to the above four questions provided for Electre Tri-C (Almeida-Dias et al., 2010, Section 3.3) can easily be transposed, without significant changes, to Electre TRI-NC. The only difference is that instead of considering a single characteristic reference action by category, several reference actions are used. This difference has no impact on the arguments given in the Electre TRI-C framework, mainly based on the manner in which the categorical credibility indices, $\sigma\left(\{a\}, B_{h}\right)$ and $\sigma\left(B_{h},\{a\}\right)$, have been defined (see Section 2.1).

As for a similar function in the Electre TRI-C framework, the selecting function (1) fulfills Properties 1 and 2 (for more details, see Almeida-Dias et al. (2010, Section 3.3 and Appendix A.1)). This proof remains valid in the ELECTRE TRI-NC framework, since it depends on the monotonicity properties of credibility indices, $\sigma\left(a, b_{h}\right)$ and $\sigma\left(b_{h}, a\right)$, which do not change with respect to $\sigma\left(\{a\}, B_{h}\right)$ and $\sigma\left(B_{h},\{a\}\right)$ (see also Appendix B). However, we did not prove that such a selecting function, defined as function (1), is the only one that fulfills Properties 1 and 2. This special issue remains as an open problem.

\subsection{Electre Tri-nC versus Electre Tri-C}

In this section, we show that Electre TRI-NC is not a simple extension, or generalization of Electre TRI-C, but it comprises several new and important features. Electre TrI-NC gives a particular freedom to the decision maker in the co-construction decision aiding process with the analyst to characterize the categories. This freedom is indeed a real advantage for the decision maker. Thus,

(1) In Electre Tri-C, the decision maker is required to conceive a unique reference action, which should be typical of the actions she/he judges appropriate to be assigned to each category according to Assumption 2. In ELECTRE TRI-NC, there is an important difference: the decision maker can introduce several representative reference actions that she/he considers as appropriate to be assigned to each category. They are not necessarily typical. In particular, she/he can introduce two reference actions, or some pairs of reference actions of the following type: $b_{h}^{t}$ and $b_{h}^{t+1}$, such that $g_{j}\left(b_{h}^{t}\right)$ is better than $g_{j}\left(b_{h}^{t+1}\right)$ for all $g_{j}$ in a subset of criteria $E \subset F$ and $g_{j}\left(b_{h}^{t+1}\right)$ is better than $g_{j}\left(b_{h}^{t}\right)$ for all $g_{j}$ not in $E \subset F$. 
(2) As in Electre Tri-C, Electre Tri-nC does not require to define a frontier between two consecutive categories, $C_{h}$ and $C_{h+1}$. However, Electre TrI-NC offers the possibility of "approaching" such a frontier. This is done by defining at least a lower characteristic reference action, $b_{h+1}^{s}$, which is judged rather closed to the "lower part" of the category $C_{h+1}$ and at least an upper characteristic reference action, $b_{h}^{r}$, which is judged rather closed to the "upper part" of the category $C_{h}$. The analyst should call the attention to the decision maker to the fact that if she/he introduces a reference action as $b_{h+1}^{s}$, such an action will be processed by ELECTRE TRI-NC as closed to the lower part of $C_{h+1}$ only when another characteristic reference action is conjointly introduced to be closed to the upper part of $C_{h+1}$.

(3) In the case where the decision maker wants to merge two consecutive categories with ELECTRE TRI-C she/he cannot keep the two characteristic reference actions that define the categories to be merged. In such a case, the decision maker may keep only one of them, or build a new one. On the contrary, when using Electre TrI-NC the decision maker can simply keep the union of the characteristic reference actions of the two merged categories in order to define the new category, which is a very natural way for doing a merging operation.

Let us notice that the splitting operation can also give an advantage to Electre Tri-NC in comparison to Electre Tri-C if it is possible to make an ordered partition of the reference actions characterizing the category to be split into two new consecutive categories, such that the dominance condition will be verified with respect to these new categories.

\section{Properties of Electre Tri-nC}

This section presents the main results based on the the structural requirements defined in Section 2.2 and the construction of the range of categories provided by ELECTRE TRI-NC as well as the impact of a new characterization of the set of categories.

\subsection{Main results}

Theorem 1(a) will bring to light the role played by a minimum required level of credibility, $\lambda^{b}$, which is defined as follows:

$$
\lambda^{b}=\max _{h=1, \ldots,(q-1)}\left\{\sigma\left(b_{h}^{r}, b_{h+1}^{s}\right), r=1, \ldots, m_{h} ; s=1, \ldots, m_{h+1}\right\} .
$$

If the hyper-strict separability condition is fulfilled, then $\lambda^{b}=0$; if the strict separability condition is fulfilled, then $\lambda^{b} \in\left[0, \frac{1}{2}\left[\right.\right.$; and, if the weak separability condition is fulfilled, then $\lambda^{b} \in[0,1[$ (see also Section 2.1).

Theorem 1. The Electre TRI-NC assignment procedure fulfills:

(a) the conformity property if $\lambda>\lambda^{b}$.

(b) the homogeneity, the monotonicity, and the stability properties.

The proof of Theorem 1 is provided in Appendix A.

Let $\lambda$ be the chosen credibility level used to define the $\lambda$-binary relations (see Definition 2, in Section 2.1). Proposition 1 presents a useful result when comparing an action $a$ to the subsets of reference actions in order to shed light on the interpretation of the assignment results provided by Electre TrI-NC (see also Theorem 2).

Proposition 1. For any action a compared to the subsets of reference actions $B_{h}$ one and only one of the three following cases occurs:

(a) Action a is neither $\lambda$-indifferent nor $\lambda$-incomparable to $B_{h}, h=1, \ldots, q$.

(b) Action a is $\lambda$-indifferent to at least one subset of reference actions $B_{h}$. Moreover, if $B_{h}$ is not unique, then the subsets of reference actions, which are $\lambda$-indifferent to action a, define a subset of consecutive categories.

(c) Action a is $\lambda$-incomparable to at least one subset of reference actions $B_{h}$. Moreover, if $B_{h}$ is not unique, then the subsets of reference actions, which are $\lambda$-incomparable to action a, define a subset of consecutive categories.

The proof of Proposition 1 is provided in Appendix B.

Theorem 2. Let $\Gamma(a)$ denote the range of consecutive categories provided by ELECTRE TRI-NC as possible categories to which an action a can be assigned to.

(a) When a is neither $\lambda$-indifferent nor $\lambda$-incomparable to $B_{h}, h=1, \ldots, q$ : $\Gamma(a)$ is composed of one or two consecutive categories. 
(b) When a is $\lambda$-indifferent to at least one subset of reference actions $B_{h}$ : $\Gamma(a)$ is composed of the subset of consecutive categories defined by such $\lambda$-indifference, and, possibly, by including one or two of the adjacent categories to them.

(c) When a is $\lambda$-incomparable to at least one subset of reference actions $B_{h}$ : $\Gamma(a)$ is composed of the subset of consecutive categories defined by such $\lambda$-incomparability, and, possibly, by including one or two of the adjacent categories to them.

The proof of Theorem 2 can easily be derived from Almeida-Dias et al. (2010, Appendix A.4) by replacing the classical credibility indices by the categorical credibility indices and based on Proposition 1. Based on this proof, let us notice that:

- In the case of $\lambda$-indifference, the descending pre-selected category is the highest category, $C_{t}$, such that $a$ is $\lambda$ indifferent to $B_{t}$, while the ascending pre-selected category is the lowest category, $C_{k}$, such that $a$ is $\lambda$-indifferent to $B_{k}$.

- In the case of $\lambda$-incomparability, the descending pre-selected category is the the worst adjacent category, $C_{t-1}$, to the lowest category, $C_{t}$, such that $a$ is $\lambda$-incomparable to $B_{t}$, while the ascending pre-selected category is the best adjacent category, $C_{k+1}$, to the highest category, $C_{k}$, such that $a$ is $\lambda$-incomparable to $B_{k}$.

\subsection{Adding or removing a characteristic reference action}

The merging and the splitting operations are two ways for modifying the characterization of the categories, which necessarily change their number. This section is devoted to another way for modifying such a characterization, which does not change their number, but at least the characterization of one of them is changed. This modification consists of adding or removing a characteristic reference action to or from a subset of reference actions. Moreover, changing the characterization of a category has also an impact on the actions which could be assigned to the two adjacent categories.

Let $b_{h}^{*}$ be a new reference action, which is added to $B_{h}$, for a new characterization of category $C_{h}$. Therefore, let $B_{h}^{*}=B_{h} \cup\left\{b_{h}^{*}\right\}$ be the modified subset of reference actions of $C_{h}$. Proposition 2 presents the impact of such a modification on the categorical credibility indices.

Proposition 2. When adding a characteristic reference action, $b_{h}^{*}$, to $B_{h}$, the new categorical credibility indices are related to the former ones as follows:

$$
\sigma\left(\{a\}, B_{h}^{*}\right) \geqslant \sigma\left(\{a\}, B_{h}\right) \text { and } \sigma\left(B_{h}^{*},\{a\}\right) \geqslant \sigma\left(B_{h},\{a\}\right)
$$

The proof of Proposition 2 is provided in Appendix C. Let us notice that the decision maker can be interested in such a modification, for instance, when she/he is not in agreement with the assignment of some actions $a$. This can mainly occur in the two following cases:

- $a$ is assigned to $C_{h-1}$, while the decision maker considers that $a$ should be assigned to $C_{h}$;

- $a$ is assigned to $C_{h+1}$, while the decision maker considers that $a$ should be assigned to $C_{h}$;

In the above two cases, the decision maker, in the co-construction interactive process with the analyst, can examine the possibility of adding such an action $a$ as an additional characteristic reference action of $C_{h}$.

Theorem 3 introduces the conditions in which such a modification can be done as well as the impact on the assignment results provided by the ELECTRE TRI-NC method.

Theorem 3. Let $b_{h}^{*}$ denote an action, which is added to $B_{h}$ as a reference action for modifying the characterization of $C_{h}$. Assume that $B^{*}=B \cup\left\{b_{h}^{*}\right\}$ fulfills at least the weak separability condition and the previous chosen credibility level, $\lambda$, verifies $\lambda>\lambda^{b^{*}}$. Let $T_{h}=\left\{C_{h-1}, C_{h}, C_{h+1}\right\}$ denote the triplet of categories, including the modified one and the two adjacent ones. Let $\Gamma(a)$ and $\Gamma^{*}(a)$ be the assignment results of ELECTRE TRI-NC before and after such modifications, respectively. The following cases occur:

(a) If both lower and upper bounds of $\Gamma(a)$ are not in $T_{h}$, then $\Gamma^{*}(a)$ remains the same as $\Gamma(a)$.

(b) If only the lower bound of $\Gamma(a)$ is in $T_{h}$ (where the upper bound is at least $C_{h+2}$ ), then either $\Gamma^{*}(a)$ remains the same as $\Gamma(a)$, or $\Gamma^{*}(a)$ is obtained from $\Gamma(a)$ by adding or removing one or both categories among $C_{h-1}$ and $C_{h}$.

(c) If only the upper bound of $\Gamma(a)$ is in $T_{h}$ (where the lower bound is at most $C_{h-2}$ ), then either $\Gamma^{*}(a)$ remains the same as $\Gamma(a)$, or $\Gamma^{*}(a)$ is obtained from $\Gamma(a)$ by adding or removing one or both categories among $C_{h}$ and $C_{h+1}$. 
(d) If both lower and upper bounds of $\Gamma(a)$ are in $T_{h}$, then either $\Gamma^{*}(a)$ remains the same as $\Gamma(a)$, or $\Gamma^{*}(a)$ is obtained from $\Gamma(a)$ by adding or removing one or two new categories from $T_{h}$, or by replacing the unique category by one or two categories from $T_{h}$, or by replacing two categories by only one from $T_{h}$.

The proof of Theorem 3 is provided in Appendix D.

Let us notice that removing a characteristic reference action, $\bar{b}_{h}$, from a subset of reference actions, $B_{h}$, is also a way of modifying the characterization of category $C_{h}$. However, it seems to us that there is less practical concerns about this modification than the above one. When considering the case of removing a characteristic reference action, the only required condition is that $B_{h}$ must contain at least two characteristic reference actions before modification. Let $\bar{\Gamma}(a)$ and $\bar{\Gamma}^{*}(a)$ be the assignment results of ELECTRE TRI-NC before and after a modification (removing), respectively. The modification of the assignment results provided by Theorem 3 is also applied is this case by substituting $\Gamma(a)$ (adding) by $\bar{\Gamma}^{*}(a)$ (removing) and $\Gamma^{*}(a)$ (adding) by $\bar{\Gamma}(a)$ (removing).

\section{A numerical example}

The aim of this section is to illustrate how the ElECTRE TRI-NC method can be used by a decision maker (DM), including the analysis of the impact of adding reference actions leading to a new characterization of some categories and the effects of splitting and merging operations.

Consider a DM who has to organize the whole process by which a large number of projects will be examined and finally implemented or rejected. She/he considers that a first step of this process could consist of making use of a sorting procedure like Electre TrI-NC in order to allocate the projects to different categories. For this purpose, the DM thinks that the following 5 categories could be appropriate: excellent $\left(C_{5}\right)$, $\operatorname{good}\left(C_{4}\right)$, moderate $\left(C_{3}\right)$, weak $\left(C_{2}\right)$, and bad $\left(C_{1}\right)$. This chosen set of categories may play a relevant role in the organizing process, since the DM considers a priori that:

- the projects assigned to $C_{1}$ should be eliminated without any further exams, while the ones assigned to $C_{5}$ should be implemented without any further exams.

- three working groups must be formed, where each one of them examines the projects assigned to $C_{4}, C_{3}$, and $C_{2}$ in order to define in what conditions (need of additional information, minor revision, major revision, and so on) such projects could be implemented or rejected.

Suppose that each project has been evaluated on a coherent set of 7 criteria. The chosen set of parameters is presented in Table 1. Each one of these criteria is to be maximized within the range $[0,100]$.

Table 1: Criteria and parameters

\begin{tabular}{crrrrrrr}
\hline & Criteria & & & & \\
\cline { 2 - 7 } Parameters & $g_{1}$ & $g_{2}$ & $g_{3}$ & $g_{4}$ & $g_{5}$ & $g_{6}$ & $g_{7}$ \\
\hline$w_{j}$ & 0.20 & 0.15 & 0.10 & 0.10 & 0.10 & 0.15 & 0.20 \\
$q_{j}$ & 5 & 5 & 5 & 5 & 5 & 5 & 5 \\
$p_{j}$ & 10 & 10 & 10 & 10 & 10 & 10 & 10 \\
$v_{j}$ & 35 & 35 & & & & 35 & 35 \\
\hline
\end{tabular}

Taking into account the manner of processing each project assigned to a given category, the DM proposes to characterize the set of categories on the following basis (see also Table 2):

- an excellent project (belonging to $C_{5}$ ) can be characterized by the performances of 95 on all the criteria.

- a good project (belonging to $C_{4}$ ) can be characterized by the performances of 85 on the first 4 criteria or on the last 4 criteria, and at least 70 on the remaining 3 criteria. Moreover, a project with the performances of 75 on all the criteria could be representative of the lower part of this category.

- a moderate project (belonging to $C_{3}$ ) can be characterized by the performances of 65 on all the criteria as representative of the upper part, and 40 on all the criteria as representative of the lower part.

- a weak project (belonging to $C_{2}$ ) can be characterized by the performances of 30 on all the criteria as representative of the upper part of this category, and 20 on all the criteria characterizes a very weak project, which could remain in the category of weak projects for excluding a direct elimination without further exams. 
- a bad project (belonging to $C_{1}$ ) can be characterized by the performances zof 5 on all the criteria.

This set of characteristic reference actions fulfills the strict separability condition, since $\lambda^{b}=0.45$. Thus, the chosen credibility level, $\lambda$, can be any value within the range $[0.5,1]$.

Table 2: Characteristic reference actions

\begin{tabular}{rrrrrrrrr}
\hline & \multicolumn{1}{c}{ Criteria } \\
\cline { 3 - 8 }$B_{h}$ & $b_{h}^{r}$ & $g_{1}$ & $g_{2}$ & $g_{3}$ & $g_{4}$ & $g_{5}$ & $g_{6}$ & $g_{7}$ \\
\hline$B_{1}$ & $b_{1}^{1}$ & 5 & 5 & 5 & 5 & 5 & 5 & 5 \\
$B_{2}$ & $b_{2}^{1}$ & 20 & 20 & 20 & 20 & 20 & 20 & 20 \\
& $b_{2}^{2}$ & 30 & 30 & 30 & 30 & 30 & 30 & 30 \\
$B_{3}$ & $b_{3}^{1}$ & 40 & 40 & 40 & 40 & 40 & 40 & 40 \\
& $b_{3}^{2}$ & 65 & 65 & 65 & 65 & 65 & 65 & 65 \\
$B_{4}$ & $b_{4}^{1}$ & 70 & 70 & 70 & 85 & 85 & 85 & 85 \\
& $b_{4}^{2}$ & 85 & 85 & 85 & 85 & 70 & 70 & 70 \\
& $b_{4}^{3}$ & 75 & 75 & 75 & 75 & 75 & 75 & 75 \\
$B_{5}$ & $b_{5}^{1}$ & 95 & 95 & 95 & 95 & 95 & 95 & 95 \\
\hline
\end{tabular}

When choosing a particular credibility level, $\lambda$, the DM may present the following reasons: when there is no veto, an outranking assertion must only be validated if at least one of the two most important criteria, $g_{1}$ or $g_{7}$, is concordant. Thus, if only one of these criteria is concordant, then for validating such an assertion it is necessary that at least 4 more criteria are concordant. If $g_{1}$ and $g_{7}$ are both concordant, then for validating such an assertion it is sufficient that at least 2 more criteria, including $g_{2}$ or $g_{6}$, will be concordant too. This implies that $0.60<\lambda \leqslant 0.65$. Following these arguments, let us suppose that the DM validates $\lambda=0.65$. Based on this chosen credibility level, let us analyse the reference actions which characterize the category $C_{4}$. Among them, $b_{4}^{1}$ and $b_{4}^{2}$ are $\lambda$-incomparable, since $\sigma\left(b_{4}^{1}, b_{4}^{2}\right)=\sigma\left(b_{4}^{2}, b_{4}^{1}\right)=0.55$. These two reference actions are both $\lambda$-preferred to $b_{4}^{3}$, since $\sigma\left(b_{4}^{1}, b_{4}^{3}\right)=\sigma\left(b_{4}^{2}, b_{4}^{3}\right)=1$ and $\sigma\left(b_{4}^{3}, b_{4}^{1}\right)=\sigma\left(b_{4}^{3}, b_{4}^{2}\right)=0.45$.

Before the final choice of the elements of the above sorting model (mainly the subset of characteristic reference actions and the chosen credibility level), the DM could examine the manner in which such a sorting model could assign some test-projects (potential actions). These projects can be fictitious, but having easily interpretable performances on the set of criteria. For such projects, the DM can a priori give an opinion concerning the category, or the range of categories, to which they can be assigned to if such projects could be effective ones. For this didactical example, the DM considers 24 test-projects (see Table 3). These test-projects could allow to revise the manner of the a priori characterization of the set of categories, the chosen credibility level, or the initial judgment regarding the assignment of them.

The categorical credibility indices of the comprehensive outranking of the potential actions over the subset of reference actions, and vice-versa, as well as the $\lambda$-binary relations, are presented in Table 4 . The assignment results provided by the ELECTRE TRI-NC method are presented in Table 5. 
Table 3: Set of test-projects (potential actions)

\begin{tabular}{|c|c|c|c|c|c|c|c|c|}
\hline \multirow[b]{2}{*}{ Actions } & \multicolumn{7}{|c|}{ Criteria } & \multirow[b]{2}{*}{ DM } \\
\hline & $g_{1}$ & $g_{2}$ & $g_{3}$ & $g_{4}$ & $g_{5}$ & $g_{6}$ & $g_{7}$ & \\
\hline$a_{1}$ & 10 & 10 & 10 & 10 & 10 & 10 & 10 & $C_{1}$ \\
\hline$a_{2}$ & 10 & 20 & 20 & 10 & 20 & 20 & 10 & $C_{1}$ \\
\hline$a_{3}$ & 15 & 5 & 10 & 15 & 10 & 5 & 15 & $C_{1}$ \\
\hline$a_{4}$ & 15 & 15 & 15 & 15 & 15 & 15 & 15 & {$\left[C_{1}, C_{2}\right]$} \\
\hline$a_{5}$ & 20 & 20 & 50 & 50 & 50 & 20 & 20 & $C_{2}$ \\
\hline$a_{6}$ & 30 & 30 & 45 & 45 & 45 & 30 & 30 & $C_{2}$ \\
\hline$a_{7}$ & 5 & 50 & 50 & 50 & 50 & 50 & 90 & {$\left[C_{2}, C_{3}\right]$} \\
\hline$a_{8}$ & 35 & 35 & 35 & 35 & 35 & 35 & 35 & {$\left[C_{2}, C_{3}\right]$} \\
\hline$a_{9}$ & 25 & 25 & 25 & 50 & 50 & 50 & 50 & {$\left[C_{2}, C_{3}\right]$} \\
\hline$a_{10}$ & 30 & 30 & 30 & 40 & 40 & 40 & 40 & $C_{3}$ \\
\hline$a_{11}$ & 30 & 45 & 45 & 45 & 45 & 45 & 30 & $C_{3}$ \\
\hline$a_{12}$ & 35 & 35 & 35 & 45 & 45 & 45 & 45 & $C_{3}$ \\
\hline$a_{13}$ & 35 & 35 & 35 & 70 & 70 & 70 & 70 & $C_{3}$ \\
\hline$a_{14}$ & 45 & 45 & 30 & 30 & 30 & 45 & 45 & $C_{3}$ \\
\hline$a_{15}$ & 65 & 25 & 25 & 25 & 25 & 25 & 65 & $C_{3}$ \\
\hline$a_{16}$ & 85 & 85 & 50 & 50 & 50 & 15 & 15 & $C_{3}$ \\
\hline$a_{17}$ & 65 & 65 & 85 & 85 & 85 & 65 & 65 & {$\left[C_{3}, C_{4}\right]$} \\
\hline$a_{18}$ & 70 & 70 & 70 & 70 & 70 & 70 & 70 & {$\left[C_{3}, C_{4}\right]$} \\
\hline$a_{19}$ & 70 & 70 & 70 & 95 & 95 & 95 & 95 & $C_{4}$ \\
\hline$a_{20}$ & 75 & 75 & 75 & 80 & 80 & 80 & 80 & $C_{4}$ \\
\hline$a_{21}$ & 80 & 80 & 80 & 80 & 80 & 80 & 80 & $C_{4}$ \\
\hline$a_{22}$ & 85 & 50 & 85 & 85 & 85 & 50 & 85 & $C_{4}$ \\
\hline$a_{23}$ & 75 & 75 & 75 & 95 & 95 & 95 & 95 & {$\left[C_{4}, C_{5}\right]$} \\
\hline$a_{24}$ & 90 & 90 & 80 & 80 & 80 & 90 & 90 & $C_{5}$ \\
\hline
\end{tabular}

Table 4: Comparison of actions

\begin{tabular}{|c|c|c|c|c|c|c|c|c|c|c|c|c|c|c|c|}
\hline \multirow[b]{2}{*}{ Actions } & \multicolumn{5}{|c|}{$\sigma\left(\{a\}, B_{h}\right)$} & \multicolumn{5}{|c|}{$\sigma\left(B_{h},\{a\}\right)$} & \multicolumn{5}{|c|}{$\lambda$-binary relations $(\lambda=0.65)^{*}$} \\
\hline & $B_{1}$ & $B_{2}$ & $B_{3}$ & $B_{4}$ & $B_{5}$ & $B_{1}$ & $B_{2}$ & $B_{3}$ & $B_{4}$ & $B_{5}$ & $B_{1}$ & $B_{2}$ & $B_{3}$ & $B_{4}$ & $B_{5}$ \\
\hline$a_{1}$ & 1.00 & 0.00 & 0.00 & 0.00 & 0.00 & 1.00 & 1.00 & 1.00 & 1.00 & 1.00 & $I$ & $P^{\prime}$ & $P^{\prime}$ & $P^{\prime}$ & $P^{\prime}$ \\
\hline$a_{2}$ & 1.00 & 0.50 & 0.00 & 0.00 & 0.00 & 0.50 & 1.00 & 1.00 & 1.00 & 1.00 & $P$ & $P^{\prime}$ & $P^{\prime}$ & $P^{\prime}$ & $P^{\prime}$ \\
\hline$a_{3}$ & 1.00 & 0.50 & 0.00 & 0.00 & 0.00 & 0.50 & 1.00 & 1.00 & 1.00 & 1.00 & $P$ & $P^{\prime}$ & $P^{\prime}$ & $P^{\prime}$ & $P^{\prime}$ \\
\hline$a_{4}$ & 1.00 & 1.00 & 0.00 & 0.00 & 0.00 & 0.00 & 1.00 & 1.00 & 1.00 & 1.00 & $P$ & $I$ & $P^{\prime}$ & $P^{\prime}$ & $P^{\prime}$ \\
\hline$a_{5}$ & 1.00 & 1.00 & 0.16 & 0.00 & 0.00 & 0.00 & 0.70 & 1.00 & 1.00 & 1.00 & $P$ & $I$ & $P^{\prime}$ & $P^{\prime}$ & $P^{\prime}$ \\
\hline$a_{6}$ & 1.00 & 1.00 & 0.30 & 0.00 & 0.00 & 0.00 & 0.70 & 1.00 & 1.00 & 1.00 & $P$ & $I$ & $P^{\prime}$ & $P^{\prime}$ & $P^{\prime}$ \\
\hline$a_{7}$ & 1.00 & 0.80 & 0.00 & 0.00 & 0.00 & 0.00 & 0.00 & 0.80 & 1.00 & 1.00 & $P$ & $P$ & $P^{\prime}$ & $P^{\prime}$ & $P^{\prime}$ \\
\hline$a_{8}$ & 1.00 & 1.00 & 1.00 & 0.00 & 0.00 & 0.00 & 1.00 & 1.00 & 1.00 & 1.00 & $P$ & $I$ & $I$ & $P^{\prime}$ & $P^{\prime}$ \\
\hline$a_{9}$ & 1.00 & 1.00 & 0.55 & 0.00 & 0.00 & 0.00 & 0.45 & 1.00 & 1.00 & 1.00 & $P$ & $P$ & $P^{\prime}$ & $P^{\prime}$ & $P^{\prime}$ \\
\hline$a_{10}$ & 1.00 & 1.00 & 0.55 & 0.00 & 0.00 & 0.00 & 0.45 & 1.00 & 1.00 & 1.00 & $P$ & $P$ & $P^{\prime}$ & $P^{\prime}$ & $P^{\prime}$ \\
\hline$a_{11}$ & 1.00 & 1.00 & 0.60 & 0.00 & 0.00 & 0.00 & 0.40 & 1.00 & 1.00 & 1.00 & $P$ & $P$ & $P^{\prime}$ & $P^{\prime}$ & $P^{\prime}$ \\
\hline$a_{12}$ & 1.00 & 1.00 & 1.00 & 0.00 & 0.00 & 0.00 & 0.45 & 1.00 & 1.00 & 1.00 & $P$ & $P$ & $I$ & $P^{\prime}$ & $P^{\prime}$ \\
\hline$a_{13}$ & 1.00 & 1.00 & 1.00 & 0.00 & 0.00 & 0.00 & 0.00 & 1.00 & 1.00 & 1.00 & $P$ & $P$ & $I$ & $P^{\prime}$ & $P^{\prime}$ \\
\hline$a_{14}$ & 1.00 & 1.00 & 0.70 & 0.00 & 0.00 & 0.00 & 0.30 & 1.00 & 1.00 & 1.00 & $P$ & $P$ & $I$ & $P^{\prime}$ & $P^{\prime}$ \\
\hline$a_{15}$ & 1.00 & 1.00 & 0.40 & 0.00 & 0.00 & 0.00 & 0.00 & 1.00 & 1.00 & 1.00 & $P$ & $P$ & $P^{\prime}$ & $P^{\prime}$ & $P^{\prime}$ \\
\hline$a_{16}$ & 1.00 & 1.00 & 0.65 & 0.00 & 0.00 & 0.00 & 0.00 & 0.65 & 1.00 & 1.00 & $P$ & $P$ & $I$ & $P^{\prime}$ & $P^{\prime}$ \\
\hline$a_{17}$ & 1.00 & 1.00 & 1.00 & 0.65 & 0.00 & 0.00 & 0.00 & 0.70 & 0.90 & 1.00 & $P$ & $P$ & $I$ & $I$ & $P^{\prime}$ \\
\hline$a_{18}$ & 1.00 & 1.00 & 1.00 & 1.00 & 0.00 & 0.00 & 0.00 & 1.00 & 1.00 & 1.00 & $P$ & $P$ & $I$ & $I$ & $P^{\prime}$ \\
\hline$a_{19}$ & 1.00 & 1.00 & 1.00 & 1.00 & 0.43 & 0.00 & 0.00 & 0.06 & 0.45 & 1.00 & $P$ & $P$ & $P$ & $P$ & $P^{\prime}$ \\
\hline$a_{20}$ & 1.00 & 1.00 & 1.00 & 1.00 & 0.00 & 0.00 & 0.00 & 0.00 & 1.00 & 1.00 & $P$ & $P$ & $P$ & $I$ & $P^{\prime}$ \\
\hline$a_{21}$ & 1.00 & 1.00 & 1.00 & 1.00 & 0.00 & 0.00 & 0.00 & 0.00 & 1.00 & 1.00 & $P$ & $P$ & $P$ & $I$ & $P^{\prime}$ \\
\hline$a_{22}$ & 1.00 & 1.00 & 1.00 & 0.70 & 0.00 & 0.00 & 0.00 & 0.22 & 0.70 & 1.00 & $P$ & $P$ & $P$ & $I$ & $P^{\prime}$ \\
\hline$a_{23}$ & 1.00 & 1.00 & 1.00 & 1.00 & 0.55 & 0.00 & 0.00 & 0.00 & 0.45 & 1.00 & $P$ & $P$ & $P$ & $P$ & $P^{\prime}$ \\
\hline$a_{24}$ & 1.00 & 1.00 & 1.00 & 1.00 & 0.70 & 0.00 & 0.00 & 0.00 & 0.55 & 1.00 & $P$ & $P$ & $P$ & $P$ & $I$ \\
\hline
\end{tabular}

${ }^{*} P=P^{\lambda}, I=I^{\lambda}, R=R^{\lambda}, a_{i} P^{\prime} B_{h}=B_{h} P a_{i}$. 


\begin{tabular}{ccc} 
Table 5: Assignment results & $(\lambda=0.65)$ \\
\hline Actions & ELECTRE TRI-NC & DM \\
\hline$a_{1}$ & $C_{1}$ & $C_{1}$ \\
$a_{2}$ & {$\left[C_{1}, C_{2}\right]$} & $C_{1}$ \\
$a_{3}$ & {$\left[C_{1}, C_{2}\right]$} & $C_{1}$ \\
$a_{4}$ & $C_{2}$ & {$\left[C_{1}, C_{2}\right]$} \\
$a_{5}$ & $C_{2}$ & $C_{2}$ \\
$a_{6}$ & $C_{2}$ & $C_{2}$ \\
$a_{7}$ & {$\left[C_{2}, C_{3}\right]$} & {$\left[C_{2}, C_{3}\right]$} \\
$a_{8}$ & {$\left[C_{2}, C_{3}\right]$} & {$\left[C_{2}, C_{3}\right]$} \\
$a_{9}$ & $C_{3}$ & {$\left[C_{2}, C_{3}\right]$} \\
$a_{10}$ & $C_{3}$ & $C_{3}$ \\
$a_{11}$ & $C_{3}$ & $C_{3}$ \\
$a_{12}$ & $C_{3}$ & $C_{3}$ \\
$a_{13}$ & $C_{3}$ & $C_{3}$ \\
$a_{14}$ & $C_{3}$ & $C_{3}$ \\
$a_{15}$ & $C_{3}$ & $C_{3}$ \\
$a_{16}$ & $C_{3}$ & $C_{3}$ \\
$a_{17}$ & {$\left[C_{3}, C_{4}\right]$} & {$\left[C_{3}, C_{4}\right]$} \\
$a_{18}$ & {$\left[C_{3}, C_{4}\right]$} & {$\left[C_{3}, C_{4}\right]$} \\
$a_{19}$ & $C_{4}$ & $C_{4}$ \\
$a_{20}$ & $C_{4}$ & $C_{4}$ \\
$a_{21}$ & $C_{4}$ & $C_{4}$ \\
$a_{22}$ & $C_{4}$ & $C_{4}$ \\
$a_{23}$ & $C_{5}$ & {$\left[C_{4}, C_{5}\right]$} \\
$a_{24}$ & $C_{5}$ & $C_{5}$ \\
\hline & &
\end{tabular}

According to Table 5 the DM can observe that:

- the assignment results provided by Electre TRI-NC are rigorously the same as her/his a priori judgements for 19 of the 24 test-projects. Among such a subset of test-projects, ElECTRE Tri-NC recognizes a "fuzzy" position with respect to the frontier between two consecutive categories when assigning four test-projects: $a_{7}, a_{8}, a_{17}$, and $a_{18}$.

- among the 5 cases where the Electre TRI-NC assignment results differ from her/his a priori judgements, 3 of them are related to the ones in which such judgements were not well established. It concerns the test-projects $a_{4}, a_{9}$, and $a_{23}$. For each one of such projects, there was a strong hesitation between two consecutive categories. She/he remarks that Electre TRI-NC removes such an hesitation and assigns each one of them to the highest category related to such an hesitation.

- for the remaining two cases from the previous point, $a_{2}$ and $a_{3}$, on the contrary Electre TRI-NC provides the categories $C_{1}$ and $C_{2}$ as a possible assignment for such test-projects. She/he considers that these projects must be eliminated without any further exams, and, therefore, they must be assigned to $C_{1}$. The possibility of assigning $a_{2}$ and $a_{3}$ to $C_{2}$ can easily be eliminated since such two projects can be added as characteristic reference actions of $C_{1}$ (in such a case, $\lambda^{b}$ becomes 0.50 ). This adding operation has only one impact: the test-project $a_{4}$ is now assigned to the range $\left[C_{1}, C_{2}\right]$, which is the same as her/his a priori judgement.

The above results have been obtained with $\lambda=0.65$. Before the final choice of such a credibility level, the DM can wish to know the assignment results when choosing a different $\lambda$ on $] 0.60,0.65]$. In this numerical example, she/he observe that there is no change on the assignment results according to this validated range of credibility levels.

The DM, in interaction with the decision aiding analyst, can wish to examine a splitting of the category $C_{3}$, since it is possible that a large number of effective projects can be assigned to it. This operation can be applied as follows: Let $C_{3}=C_{3}^{\prime} \cup C_{3}^{\prime \prime}$. The two new categories can, for instance, be characterized as follows: $C_{3}^{\prime}$ by $B_{3}^{\prime}=\left\{b_{3}^{1^{\prime}}, b_{3}^{2^{\prime}}\right\}$, where $g_{j}\left(b_{3}^{1^{\prime}}\right)=g_{j}\left(b_{3}^{1}\right)=40$ and $g_{j}\left(b_{3}^{2^{\prime}}\right)=45, j=1, \ldots, 7$; as well as $C_{3}^{\prime \prime}$ by $B_{3}^{\prime \prime}=\left\{b_{3}^{1^{\prime \prime}}, b_{3}^{2^{\prime \prime}}\right\}$, where $g_{j}\left(b_{3}^{1^{\prime \prime}}\right)=55$ and $g_{j}\left(b_{3}^{2^{\prime \prime}}\right)=$ $g_{j}\left(b_{3}^{2}\right)=65, j=1, \ldots, 7$. After the splitting operation, when applying Electre TRI-NC with the new set of 6 categories the DM can observe that:

- the test-projects previously assigned to $C_{3}$ are either assigned to $C_{3}^{\prime}$ or to $C_{3}^{\prime \prime}$.

$$
\begin{aligned}
& \text {. } a_{9}, a_{10}, a_{11}, a_{12}, a_{14} \text {, and } a_{15} \text { are assigned to } C_{3}^{\prime} \text {; } \\
& \text {. } a_{13} \text { is assigned to } C_{3}^{\prime \prime} ; \text { and } \\
& \text {. } a_{16} \text { is assigned to }\left[C_{3}^{\prime}, C_{3}^{\prime \prime}\right] .
\end{aligned}
$$


- $a_{8}$ previously assigned to $\left[C_{2}, C_{3}\right]$ is now assigned to $\left[C_{2}, C_{3}^{\prime}\right] ; a_{7}$ previously assigned to $\left[C_{2}, C_{3}\right]$ is now assigned to $C_{3}^{\prime}$; and, $a_{17}$ and $a_{18}$ previously assigned to $\left[C_{3}, C_{4}\right]$ are now assigned to $\left[C_{3}^{\prime \prime}, C_{4}\right]$.

- the remaining test-projects are assigned to the same categories as before the splitting operation.

Based on the assignment results after the splitting operation, let us also illustrate a merging operation applied to categories $C_{2}$ and $C_{3}^{\prime}$. The new category, denoted $C_{2}^{*}$, such that $C_{2}^{*}=C_{2} \cup C_{3}^{\prime}$, can trivially be characterized by $B_{2} \cup B_{3}^{\prime}$. Let us notice that in such a set the two reference actions $b_{2}^{2}$ and $b_{3}^{1^{\prime}}$ are redundant taking into account the dominance condition. Therefore, the new category $C_{2}^{*}$ can be characterized by $B_{2}^{*}=\left\{b_{2}^{1^{*}}, b_{2}^{2^{*}}\right\}$, where $g_{j}\left(b_{2}^{1^{*}}\right)=g_{j}\left(b_{2}^{1}\right)=20, j=1, \ldots, 7$; and $g_{j}\left(b_{2}^{2^{*}}\right)=g_{j}\left(b_{3}^{2^{\prime}}\right)=45, j=1, \ldots, 7$. After the merging operation, when applying ELECTRE TRI-NC with the new set of 5 categories the DM can observe that:

- the test-projects previously assigned to $C_{2}$ and $C_{3}^{\prime}$ are, after the merging of these two categories, assigned to the new category, $C_{2}^{*}$, excluding $a_{7}$, which is now assigned to the range $\left[C_{2}^{*}, C_{3}^{\prime \prime}\right]$.

- the test-projects $a_{4}$ and $a_{16}$ remain assigned to the same range of two consecutive categories, i.e. $\left[C_{1}, C_{2}^{*}\right]$ and $\left[C_{2}^{*}, C_{3}^{\prime \prime}\right]$, respectively.

- the remaining ones are assigned to the same categories as before the merging operation.

The two above operations of splitting and merging put in light the stability of the assignment obtained with ELECTRE TRI-NC.

\section{Comparison with related sorting methods}

The aim of this section is to examine several sorting methods, where characteristic reference actions are initially used for representing the willing of the decision maker about the assignment to each category. Two different decision aiding sorting contexts must be distinguished.

The first one is the case where the set of categories is unordered and each category is characterized by the same kind of reference actions as used in ELECTRE TRI-NC. See, for instance, the filtering by indifference assignment procedure, denoted here FIP (Perny, 1998), the most indifferent prototype assignment procedure, denoted PIP (Henriet, 2000), the ProAFTN assignment procedure (Belacel, 2000), the TRINOMFC assignment procedure (Léger and Martel, 2002), and the sorting by preference closeness assignment procedure, denoted here Closort (Fernández et al., 2008, 2009). Electre Tri-NC differs from these sorting methods, since it is applied to sorting contexts where the set of categories is completely ordered; it is based on a strong relationship between the preference direction of the criteria and the preference direction of the categories, while this relationship does not necessarily exist in the above sorting methods; and it is founded on credibility degrees of an outranking relation instead of a closeness relation, or a similar relation.

The second decision aiding context is the one where the set of categories is completely ordered. Here two different cases must be distinguished. The first of these two cases is the one where the categories are characterized by boundary reference actions. In such a case, some sorting methods are based on flows like in the PrometheE methodology. See, for instance, the FlowSort assignment procedure (Nemery and Lamboray, 2008), the PAIRClas assignment procedure (Doumpos and Zopounidis, 2004), and the PromSort assignment procedure (Araz and Ozkarahan, 2007). There are also some sorting methods based on the ELECTRE methodology. See, for instance, the generalized conjunctive assignment procedure, denoted here FSY (Yu, 1992, pp. 108-141), the filtering by strict preference assignment procedure, denoted here FPP (Perny, 1998), and the fuzzy inclusion assignment procedure, denoted here FIS (Rigopoulos et al., 2008).

The second case deals with methods where a set of reference actions is a priori proposed by the decision maker on the basis of a co-construction interactive process with the analyst; each of the reference actions being supposed to be representative or informative of the actions that should be assigned to a given category. Besides the ElECTRE TRI-NC framework, this case was already considered in several published works.

Let us mention the following ones: Greco et al. (2002), Błaszczyński et al. (2007), and Dembczyński et al. (2009). The sorting procedures presented in these works are theoretically based on new developments of the rough set theory. Their aim is to propose to the decision maker a set of decision rules for the assignment of the actions to the categories.

The UTADIS ${ }^{\text {GMS }}$ method by Greco et al. (2010) consists of building a set of instances of a preference model that restores the assignment of the reference actions in the most adequate way according to the decision maker wishes (expressed as preference statements provided by the decision maker). A fundamental difference with Electre TRI-NC comes from fact 
that the preference model is built on the construction of an additive value function and not on one or several outranking relations as it is the case in Electre type methods. Besides this fundamental difference, the authors introduce and distinguish two concepts: a necessary assignment and a possible assignment.

The Theseus method (Fernández and Navarro, 2011) must be considered here for a brief comparison with ElectRE TRI-NC. This method can be considered in the framework of the second case. The four premises under which the method is based on (Fernández and Navarro, 2011, see Section 2) have some common features with the three assumptions of ELECTRE TRI-NC (see Section 1), which should be fulfilled in each decision aiding context Electre TRI-NC is used. Nevertheless, important differences exist between our Assumptions 2 and 3 and the Premises (iii) and (iv) in Fernández and Navarro (2011). Consequently, the role which are intended to play the categories in the decision aiding contexts, as well as the manner of how to define all the reference actions (used for characterize these categories) can be very different, depending on whether the analyst uses Electre Tri-NC or Theseus. Besides, this aspects, in Electre Tri-NC we do not follow the Implications (1) (Fernández and Navarro, 2011, see Section 2), which should be fulfilled by THEseus.

Consequently, the assignment procedure of ELECTRE TRI-NC is significantly different from those of Theseus. A last difference should be pointed out. In Electre TRI-NC a weak separability condition (see Condition 2 in Section 2.1) between categories should be fulfilled. It imposes some constraints for each characteristic reference action in order to guarantee the conformity with respect to the order of the categories. Such constraints do not exist in ThEseus. Based on these constraints ELECTRE TRI-NC fulfills some stability properties, which are the requirements introduced a priori (see Definition 3.d). Such stability properties are not guaranteed in Theseus. However, the absence of constraints in Theseus gives more freedom to the decision maker for the choice of the reference actions.

\section{Conclusions}

In this paper, we presented a new sorting method, called ELECTRE TRI-NC, which gives new possibilities to the DM for characterizing the categories, such that the dominance condition remains fulfilled, as in the ELECTRE TRI-C framework. When each category is characterized by a single reference actions, which is a typical reference action, then Electre TRI-NC does not differ from Electre Tri-C.

This new method, allows, in our opinion, to model a larger number of decision aiding situations in the field of sorting problems. It was proved in this paper that the ELECTRE TRI-NC method fulfills some fundamental properties: conformity, homogeneity, monotonicity, and stability.

The numerical example presented in Section 5 shows how ELECTRE TRI-NC works.

The weak separability condition has been defined by supposing that each characteristic reference actions of a category dominates each characteristic reference actions of the worst adjacent category. However, in some particular cases, this condition can be restrictive for a more flexible characterization of the set of categories. Taking into account the numerical example, the weak separability condition does not allow to add the action $a_{22}$ as characteristic reference action of the category $C_{4}$. As for future research, we will analyse in what conditions is it possible to weaken the dominance condition as it was taken into account in the weak separability condition in order to give more freedom to the DM for characterizing the set of categories. In such a case, it seem necessary to deeply analyse the conditions in which the properties of conformity and stability remain fulfilled.

We showed in the paper that adding or removing reference actions can be made interactively through the co-construction process between the DM and the decision aiding analyst. This analysis provides indeed several advantages for characterizing the set of categories.

The comparison of ELECTRE TRI-NC to some related sorting methods, using several reference actions to characterize each one of the categories, allows to conclude that the analysis provided in this paper is original and useful for sorting problems (see Section 6).

As for additional future research avenues, a decision support system incorporating the concept of characteristic reference actions is to be implemented. At the same time, we should focus our attention on the inference of some parameters through an disaggregation-aggregation elicitation techniques using characteristic reference actions.

\section{Acknowledgements}

Juscelino ALMEIDA Dias acknowledges the financial support from the Grant SFRH/ BD/ 22985/ 2005 (Fundação para a Ciência e a Tecnologia, Portugal). The authors also acknowledge the financial support from the Luso-French bilateral cooperation agreement between LAMSADE and CEG-IST (FCT/CNRS 2009). This research also partially benefited from the COST Action Grant IC0602 on "Algorithmic Decision Theory". 


\section{Appendix A. Proof of Theorem 1}

This proof is applied to the descending rule and it can easily be transposed to the ascending rule.

(a) Conformity:

Assume that the strict separability condition holds. Such a condition means that $\sigma\left(b_{h}^{r}, b_{h+1}^{s}\right)<\frac{1}{2}$, for $r=1, \ldots, m_{h} ; s=1, \ldots, m_{h+1}$; and, $h=1, \ldots,(q-1)$. This implies that for each $b_{h}^{r}$, for $r=1, \ldots, m_{h}$; and $h=1, \ldots,(q-1)$, one has $\max _{s=1, \ldots, m_{h+1}}\left\{\sigma\left(b_{h}^{r}, b_{h+1}^{s}\right)\right\}<\frac{1}{2}$. In other words, $\sigma\left(\left\{b_{h}^{r}\right\}, B_{h+1}\right)<\frac{1}{2}$, for $r=1, \ldots, m_{h}$; and $h=1, \ldots,(q-1)$. In such a case, one has $\lambda>\lambda^{b}$ for any $\lambda \in\left[\frac{1}{2}, 1\right]$. By definition of the characteristic actions and by construction of the credibility indices, one has $\sigma\left(b_{h+1}^{s}, b_{h}^{r}\right)=1$, for $s=1, \ldots, m_{h+1}$; $r=1, \ldots, m_{h}$; and, $h=1, \ldots,(q-1)$, and $\sigma\left(b_{h}^{r}, b_{h}^{r}\right)=1$, for $r=1, \ldots, m_{h}$; and $h=1, \ldots, q$. This implies that $\sigma\left(\left\{b_{h+1}^{s}\right\}, B_{h}\right)=1$, for $s=1, \ldots, m_{h+1}$; and $h=1, \ldots,(q-1)$, and $\sigma\left(\left\{b_{h}^{r}\right\}, B_{h}\right)=1$, for $r=1, \ldots, m_{h}$; and $h=1, \ldots, q$. When applying the descending rule, the pre-selected category for the characteristic action $b_{t}^{r}$ is $C_{t}$ since $\sigma\left(b_{t}, b_{t}\right)$ is always strictly greater than to any $\lambda \in\left[\frac{1}{2}, 1[\right.$ and equal to $\lambda$ if such a chosen credibility level is 1 . $C_{t}$ is selected for the assignment of each characteristic action $b_{t}^{r}$ if and only if $\rho\left(\left\{b_{t}^{r}\right\}, B_{t}\right)>\rho\left(\left\{b_{t}^{r}\right\}, B_{t+1}\right)$. This condition is verified, since $\rho\left(\left\{b_{t}^{r}\right\}, B_{t}\right)$ fulfills Property 1 . The proof is similar when $\lambda^{b} \geqslant \frac{1}{2}$.

(b) Homogeneity, monotonicity, and stability:

This part of the proof can easily be derived from Almeida-Dias et al. (2010, Appendix A.2), taking into account the role of the categorical credibility indices on the assignment process.

\section{Appendix B. Proof of Proposition 1}

This proof is trivial by proving the monotonicity of the categorical credibility degrees, $\sigma\left(\{a\}, B_{h}\right)$ and $\sigma\left(B_{h},\{a\}\right)$, and by taking into account that $B_{h}$ will play the same role as $b_{h}$ regarding Electre Tri-C (Almeida-Dias et al., 2010) and Electre Tri-B (Roy and Bouyssou, 1993, Rés. 6.3.1, p. 392) (at least in a first step).

First, consider that each subset of reference actions, $B_{h}$, has only one characteristic reference action, such that $B_{h}=\left\{b_{h}\right\}, h=$ $1, \ldots, q$. In such a case, since the set of categories is completely ordered, the following monotonicity properties are trivially verified:

(a) When at least $b_{h+1}$ strictly dominates $b_{h}, h=1, \ldots,(q-1)$, one has: $\sigma\left(a, b_{h}\right)$ is a monotonic non-increasing function of $b_{h}$, $h=1, \ldots, q$. This means that $\sigma\left(a, b_{h}\right) \geqslant \sigma\left(a, b_{h+1}\right), h=1, \ldots,(q-1)$; and, $\sigma\left(b_{h}, a\right)$ is a monotonic non-decreasing function of $b_{h}$, $h=1, \ldots, q$. This means that $\sigma\left(b_{h}, a\right) \leqslant \sigma\left(b_{h+1}, a\right), h=1, \ldots,(q-1)$.

(b) If action $a$ strictly dominates action $a^{\prime}$, then: $\sigma\left(a, b_{h}\right) \geqslant \sigma\left(a^{\prime}, b_{h}\right), h=1, \ldots, q$; and $\sigma\left(b_{h}, a\right) \leqslant \sigma\left(b_{h}, a^{\prime}\right), h=1, \ldots, q$.

Second, let us now prove the following two properties: (1) $\sigma\left(\{a\}, B_{h}\right)$ is a monotonic non-increasing function of the subsets $B_{h}$, $h=1, \ldots, q$; and (2) $\sigma\left(B_{h},\{a\}\right)$ is a monotonic non-decreasing function of the subsets $B_{h}, h=1, \ldots, q$. Therefore, one has:

(1) Consider now that each subset of reference actions, $B_{h}$, has more than one characteristic reference action. Taking into account that all the characteristic reference actions belonging to $B_{h+1}$ differ from all characteristic reference actions belonging to $B_{h}$ and all the characteristic reference actions belonging to $B_{h+1}$ are at least weakly preferred to all characteristic reference actions belonging to $B_{h}, h=1, \ldots,(q-1)$, then, according to (a) and (b) above, the following conditions are verified: $\max _{r=1, \ldots, m_{h}}\left\{\sigma\left(a, b_{h}^{r}\right)\right\}$ $\geqslant \max _{s=1, \ldots, m_{h+1}}\left\{\sigma\left(a, b_{h+1}^{s}\right)\right\}$ and $\max _{r=1, \ldots, m_{h}}\left\{\sigma\left(a, b_{h}^{r}\right)\right\} \geqslant \max _{r=1, \ldots, m_{h}}\left\{\sigma\left(a^{\prime}, b_{h}^{r}\right)\right\}$. This proves that $\sigma\left(\{a\}, B_{h}\right)$ is a monotonic nonincreasing function of the subsets $B_{h}, h=1, \ldots, q$.

(2) Consider now that each subset of reference actions, $B_{h}$, has more than one characteristic reference action. Taking into account that all the characteristic reference actions belonging to $B_{h+1}$ differ from all characteristic reference actions belonging to $B_{h}$ and all the characteristic reference actions belonging to $B_{h+1}$ are at least weakly preferred to all characteristic reference actions belonging to $B_{h}, h=1, \ldots,(q-1)$, then, according to (a) and (b) above, the following conditions are verified: $\max _{r=1, \ldots, m_{h}}\left\{\sigma\left(b_{h}^{r}, a\right)\right\}$ $\leqslant \max _{s=1, \ldots, m_{h+1}}\left\{\sigma\left(b_{h+1}^{s}, a\right)\right\}$ and $\max _{r=1, \ldots, m_{h}}\left\{\sigma\left(b_{h}^{r}, a\right)\right\} \leqslant \max _{r=1, \ldots, m_{h}}\left\{\sigma\left(b_{h}^{r}, a^{\prime}\right)\right\}$. This proves that $\sigma\left(B_{h},\{a\}\right)$ is a monotonic nondecreasing function of the subsets $B_{h}, h=1, \ldots, q$.

\section{Appendix C. Proof of Proposition 2}

Let $b_{h}^{*}$ be a characteristic reference action, which is added to $B_{h}$, such that $B_{h}^{*}=B_{h} \cup\left\{b_{h}^{*}\right\}$ is the new subset of reference actions for a new characterization of category $C_{h}$. For more details regarding the properties of the categorical credibility indices, see also Appendix B. Taking into account the definition of the credibility indices as well as their properties of monotonicity, the relationship between the categorical credibility indices (Definition 1), before and after such modification, is defined as one of the following cases:

(a) If $b_{h}^{*}$ is "comprehensively the same" as one of the characteristic reference actions from $B_{h}$, then one has $\exists r, \sigma\left(a, b_{h}^{*}\right)=\sigma\left(a, b_{h}^{r}\right)$ and $\sigma\left(b_{h}^{*}, a\right)=\sigma\left(b_{h}^{r}, a\right)$. In such a case, regarding the categorical credibility indices, the following conditions are verified: $\max _{r=1, \ldots, m_{h}}\left\{\sigma\left(a, b_{h}^{r}\right), \sigma\left(a, b_{h}^{*}\right)\right\}=\max _{r=1, \ldots, m_{h}}\left\{\sigma\left(a, b_{h}^{r}\right)\right\}$, which proves that $\sigma\left(\{a\}, B_{h}^{*}\right)=\sigma\left(\{a\}, B_{h}\right) ; \operatorname{and} \max _{s}=1, \ldots, m_{h}\left\{\sigma\left(b_{h}^{s}, a\right), \sigma\left(b_{h}^{*}, a\right)\right\}$ $=\max _{s=1, \ldots, m_{h}}\left\{\sigma\left(b_{h}^{s}, a\right)\right\}$, which proves that $\sigma\left(B_{h}^{*},\{a\}\right)=\sigma\left(B_{h},\{a\}\right)$. 
(b) If $b_{h}^{*}$ is "comprehensively better" than all the characteristic reference actions from $B_{h}$, then one has $\forall r, \sigma\left(a, b_{h}^{*}\right)<\sigma\left(a, b_{h}^{r}\right)$ and $\sigma\left(b_{h}^{*}, a\right)>\sigma\left(b_{h}^{r}, a\right)$. In such a case, regarding the categorical credibility indices, the following conditions are verified: $\max _{r=1, \ldots, m_{h}}\left\{\sigma\left(a, b_{h}^{r}\right), \sigma\left(a, b_{h}^{*}\right)\right\}=\max _{r=1, \ldots, m_{h}}\left\{\sigma\left(a, b_{h}^{r}\right)\right\}$, which proves that $\sigma\left(\{a\}, B_{h}^{*}\right)=\sigma\left(\{a\}, B_{h}\right) ;$ and $\max _{s=1, \ldots, m_{h}}\left\{\sigma\left(b_{h}^{s}, a\right), \sigma\left(b_{h}^{*}, a\right)\right\}$ $>\max _{s=1, \ldots, m_{h}}\left\{\sigma\left(b_{h}^{s}, a\right)\right\}$, which proves that $\sigma\left(B_{h}^{*},\{a\}\right)>\sigma\left(B_{h},\{a\}\right)$.

(c) If $b_{h}^{*}$ is "comprehensively worse" than all the characteristic reference actions from $B_{h}$, then one has $\forall r, \sigma\left(a, b_{h}^{*}\right)>\sigma\left(a, b_{h}^{r}\right)$ and $\sigma\left(b_{h}^{*}, a\right)<\sigma\left(b_{h}^{r}, a\right)$. In such a case, regarding the categorical credibility indices, the following conditions are verified: $\max _{r=1, \ldots, m_{h}}\left\{\sigma\left(a, b_{h}^{r}\right), \sigma\left(a, b_{h}^{*}\right)\right\}>\max _{r=1, \ldots, m_{h}}\left\{\sigma\left(a, b_{h}^{r}\right)\right\}$, which proves that $\sigma\left(\{a\}, B_{h}^{*}\right)>\sigma\left(\{a\}, B_{h}\right) ;$ and $\max _{s=1, \ldots, m_{h}}\left\{\sigma\left(b_{h}^{s}, a\right), \sigma\left(b_{h}^{*}, a\right)\right\}$ $=\max _{s=1, \ldots, m_{h}}\left\{\sigma\left(b_{h}^{s}, a\right)\right\}$, which proves that $\sigma\left(B_{h}^{*},\{a\}\right)=\sigma\left(B_{h},\{a\}\right)$.

(d) If $b_{h}^{*}$ is "comprehensively the same" as an action $a$, then, for such an action $a$, one can obtain $\exists r, \sigma\left(a, b_{h}^{*}\right)>\sigma\left(a, b_{h}^{r}\right)$ and $\sigma\left(b_{h}^{*}, a\right)>\sigma\left(b_{h}^{r}, a\right)$. Taking into account (b) and (c) above, regarding the categorical credibility indices, the following conditions can be verified: $\sigma\left(\{a\}, B_{h}^{*}\right)>\sigma\left(\{a\}, B_{h}\right)$; and $\sigma\left(B_{h}^{*},\{a\}\right)>\sigma\left(B_{h},\{a\}\right)$.

Based on the above four cases, one proves that when adding a characteristic reference actions to $B_{h}$, one necessarily obtains $\sigma\left(\{a\}, B_{h}^{*}\right) \geqslant$ $\sigma\left(\{a\}, B_{h}\right)$ and $\sigma\left(B_{h}^{*},\{a\}\right) \geqslant \sigma\left(B_{h},\{a\}\right)$

\section{Appendix D. Proof of Theorem 3}

A new reference action $b_{h}^{*}$ can only be added to $B_{h}$ for modifying the characterization of $C_{h}$, if the new set of reference actions, $B^{*}=B \cup\left\{b_{h}^{*}\right\}$, fulfills at least the weak separability condition in order to respect the meaningful ordered character of the set of categories. Additionally, the conformity property must hold in order to continue with a coherent assignment model, which means that $\lambda>\lambda^{b^{*}}$, where $\lambda$ is the same chosen credibility level before and after modification, and $\lambda^{b^{*}}$ is the minimum required credibility level associated to $B^{*}$. The aim of this proof is also to show that adding a new reference action for a new characterization of $C_{h}$, can only produce additional effects on the two adjacent categories, $C_{h-1}$ and $C_{h+1}$. For this purpose, let $T_{h}$ be a triplet of categories, such that $T_{h}=\left\{C_{h-1}, C_{h}, C_{h+1}\right\}$. Let $\Gamma(a)$ and $\Gamma^{*}(a)$ be the assignment results of ELECTRE TRI-NC before and after modification, respectively.

Taking into account the Electre TRI-NC assignment procedure, $\Gamma(a)$ can be composed according to 15 different cases within the modification framework (see Table D.6). These cases can be grouped into 4 classes: Class A, where both lower and upper bounds of $\Gamma(a)$ are not in $T_{h}$; Class B, where only the lower bound of $\Gamma(a)$ is in $T_{h}$; Class C, where only the upper bound of $\Gamma(a)$ is in $T_{h}$; Class D, where both lower and upper bounds of $\Gamma(a)$ are in $T_{h}$.

Taking into account the 4 classes identified in Table D.6, it is required to prove successively that:

I. If $\Gamma(a)$ is associated with Class $\mathrm{A}$, then $\Gamma^{*}(a)$ remains the same as $\Gamma(a)$.

II. If $\Gamma(a)$ is associated with Class $\mathrm{B}$, then one of the following cases occurs:

(1) $\Gamma^{*}(a)$ remains the same as $\Gamma(a)$.

(2) $\Gamma^{*}(a)$ is obtained from $\Gamma(a)$ by adding one or both categories among $C_{h-1}$ and $C_{h}$.

(3) $\Gamma^{*}(a)$ is obtained from $\Gamma(a)$ by removing one or both categories among $C_{h-1}$ and $C_{h}$.

III. If $\Gamma(a)$ is associated with Class $\mathrm{C}$, then one of the following cases occurs:

(1) $\Gamma^{*}(a)$ remains the same as $\Gamma(a)$.

(2) $\Gamma^{*}(a)$ is obtained from $\Gamma(a)$ by adding one or both categories among $C_{h}$ and $C_{h+1}$.

(3) $\Gamma^{*}(a)$ is obtained from $\Gamma(a)$ by removing one or both categories among $C_{h}$ and $C_{h+1}$.

IV. If $\Gamma(a)$ is associated with Class D1, then one of the following cases occurs:

(1) $\Gamma^{*}(a)$ remains the same as $\Gamma(a)$.

(2) $\Gamma^{*}(a)$ is obtained from $\Gamma(a)$ by adding one or two new categories from $T_{h}$.

(3) $\Gamma^{*}(a)$ is obtained from $\Gamma(a)$ by replacing the unique category by one or two categories from $T_{h}$.

V. If $\Gamma(a)$ is associated with Class D2, then one of the following cases occurs:

(1) $\Gamma^{*}(a)$ remains the same as $\Gamma(a)$.

(2) $\Gamma^{*}(a)$ is obtained from $\Gamma(a)$ by adding a new category from $T_{h}$.

(3) $\Gamma^{*}(a)$ is obtained from $\Gamma(a)$ by removing one or two categories from $T_{h}$.

(4) $\Gamma^{*}(a)$ is obtained from $\Gamma(a)$ by replacing two categories by only one from $T_{h}$. 
Table D.6: Cases on the range of categories

\begin{tabular}{|c|c|c|c|}
\hline Lowest category & Highest category & Case & Class \\
\hline$\geq C_{h+2}$ & $\geq C_{h+2}$ & Case 1 & Class A \\
\hline \multirow{2}{*}{$C_{h+1}$} & $\geq C_{h+2}$ & Case 2 & Class B \\
\hline & $C_{h+1}$ & Case 3 & Class D1 \\
\hline \multirow{3}{*}{$C_{h}$} & $\geq C_{h+2}$ & Case 4 & Class B \\
\hline & $C_{h+1}$ & Case 5 & Class D2 \\
\hline & $C_{h}$ & Case 6 & Class D1 \\
\hline \multirow{4}{*}{$C_{h-1}$} & $\geq C_{h+2}$ & Case 7 & Class B \\
\hline & $C_{h+1}$ & Case 8 & Class D2 \\
\hline & $C_{h}$ & Case 9 & Class D2 \\
\hline & $C_{h-1}$ & Case 10 & Class D1 \\
\hline \multirow{5}{*}{$\leq C_{h-2}$} & $\geq C_{h+2}$ & Case 11 & Class A \\
\hline & $C_{h+1}$ & Case 12 & Class C \\
\hline & $C_{h}$ & Case 13 & Class C \\
\hline & $C_{h-1}$ & Case 14 & Class C \\
\hline & $\leq C_{h-2}$ & Case 15 & Class A \\
\hline
\end{tabular}

Let us prove one case of each class:

(1) Class A. Consider the Case 1 from Table D.6. This proof is trivial since there are no changes in $B_{h+1}$ and $B_{h+2}$, which are relevant for computing $\Gamma^{*}(a)$ (Case I.). Thus, $\Gamma^{*}(a)$ remains the same as $\Gamma(a)$.

(2) The proof of Cases II.1, III.1, IV.1, and V.1 are trivial when Proposition 2 (see case (a) of its proof) is true, but it is not the only situation in which such four cases hold (see below).

(3) Class B. Consider the Case 2 from Table D.6. $\Gamma^{*}(a)$ can differ from $\Gamma(a)$ if and only if $C_{h+1}$ is obtained when the descending pre-selected is $C_{h}$, or when the ascending pre-selected category is $C_{h+1}$, before modification. It is trivial to verify that there is no change in the upper bound of $\Gamma(a)$. Taking into account Proposition 2 (see cases (b) and (c) in the proof), the following situations must be analyzed:

- After modification, the descending pre-selected category remains $C_{h}$. In such a case, one has $\sigma\left(\{a\}, B_{h}^{*}\right) \geqslant \lambda$ and $\sigma\left(\{a\}, B_{h+1}\right)<$ $\lambda$. If $\rho\left(\{a\}, B_{h}^{*}\right) \leqslant \rho\left(\{a\}, B_{h+1}\right)$, then $C_{h+1}$ remains the descending selected category for a possible assignment of $a$, which means that $\Gamma^{*}(a)$ remains the same as $\Gamma(a)$ (Case II.1); otherwise, $C_{h}$ becomes the descending selected category. In the latter case, $\Gamma^{*}(a)$ is obtained from $\Gamma(a)$ by adding the category $C_{t}$ (Case II.2).

- After modification, the ascending pre-selected category remains $C_{h+1}$. In such a case, one has $\sigma\left(B_{h}^{*},\{a\}\right)<\lambda$ and $\sigma\left(B_{h+1},\{a\}\right) \geqslant$ $\lambda$. If $\rho\left(\{a\}, B_{h+1}\right)>\rho\left(\{a\}, B_{h}^{*}\right)$, then $C_{h+1}$ remains the ascending selected category for a possible assignment of $a$, which means that $\Gamma^{*}(a)$ remains the same as $\Gamma(a)$ (Case II.1); otherwise, $C_{h}$ becomes the ascending selected category. In the latter case, $\Gamma^{*}(a)$ is obtained from $\Gamma(a)$ by adding the category $C_{k}$ (Case II.2).

- After modification, the ascending pre-selected category becomes $C_{h}$. In such a case, one has $\sigma\left(B_{h}^{*},\{a\}\right) \geqslant \lambda$ and $\sigma\left(B_{h-1},\{a\}\right)<$ $\lambda$. If $\rho\left(\{a\}, B_{h}^{*}\right)>\rho\left(\{a\}, B_{h-1}\right)$, then $C_{h}$ becomes the ascending selected category for a possible assignment of $a$; otherwise, $C_{h-1}$ becomes the ascending selected category. Thus, $\Gamma^{*}(a)$ is obtained from $\Gamma(a)$ by adding the category $C_{k}$, or both categories $C_{k}$ and $C_{h-1}$, respectively (Case II.2).

(4) Class C. Consider the Case 12 from Table D.6. The analysis of this case is similar to the one provided above for the Case 2. On the contrary, it is trivial to verify that there is no change in the lower bound of $\Gamma(a)$. Taking into account Proposition 2 (see cases (b) and (c) of its proof), the following situations must be analyzed:

- After modification, the descending pre-selected category remains $C_{h}$. When $C_{h+1}$ remains the descending selected category for a possible assignment of $a$, then $\Gamma^{*}(a)$ remains the same as $\Gamma(a)$ (Case III.1), or when $C_{h}$ becomes the descending selected category, then $\Gamma^{*}(a)$ is obtained from $\Gamma(a)$ by removing the category $C_{h+1}$ (Case III.3).

- After modification, the ascending pre-selected category remains $C_{h+1}$. When $C_{h+1}$ remains the ascending selected category for a possible assignment of $a$, then $\Gamma^{*}(a)$ remains the same as $\Gamma(a)$ (Case III.1), or when $C_{h}$ becomes the ascending selected category, then $\Gamma^{*}(a)$ is obtained from $\Gamma(a)$ by removing the category $C_{h+1}$ (Case III.3). 
- After modification, the ascending pre-selected category becomes $C_{h}$. When $C_{h}$ or $C_{h-1}$ becomes the ascending selected category for a possible assignment of $a$, then $\Gamma^{*}(a)$ is obtained from $\Gamma(a)$ by removing the category $C_{h+1}$, or both categories $C_{h}$ and $C_{h+1}$, respectively (Case III.3).

(5) Class D. Consider the Case 6 from Table D.6. $\Gamma^{*}(a)$ can differ from $\Gamma(a)$ if and only if $C_{h}$ is obtained when the descending pre-selected is $C_{h}$ and the ascending pre-selected category is $C_{h+1}$, or when the descending pre-selected is $C_{h-1}$ and the ascending pre-selected category is $C_{h}$, before modification. Taking into account Proposition 2 (see cases (b) and (c) of its proof), the following situations must be analyzed:

- After modification, the descending pre-selected category remains necessarily $C_{h}$ and the ascending pre-selected category becomes $C_{h}$. If the descending pre-selected category remains necessarily $C_{h}$, then one necessarily has $\sigma\left(\{a\}, B_{h}^{*}\right) \geqslant \lambda$, $\sigma\left(\{a\}, B_{h+1}\right)<\lambda$, and $\rho\left(\{a\}, B_{h}^{*}\right)>\rho\left(\{a\}, B_{h+1}\right)$, which imply that the descending selected category remains $C_{h}$. If the ascending pre-selected category becomes $C_{h}$, then one has $\sigma\left(B_{h}^{*},\{a\}\right) \geqslant \lambda$ and $\sigma\left(B_{h-1},\{a\}\right)<\lambda$. In such a case, if $\rho\left(\{a\}, B_{h}^{*}\right)>$ $\rho\left(\{a\}, B_{h-1}\right)$, then the ascending selected category remains $C_{h}$, which means that $\Gamma^{*}(a)=\Gamma(a)=C_{h}$ (Case IV.1); otherwise, the ascending selected category becomes $C_{h-1}$. In the latter case, $\Gamma^{*}(a)$ is obtained from $\Gamma(a)$ by adding the category $C_{h-1}$, such that $\Gamma^{*}(a)=\left[C_{h-1}, C_{h}\right]$ (Case IV.2).

- After modification, the descending pre-selected category becomes $C_{h}$ and the ascending pre-selected category remains necessarily $C_{h}$. If the ascending pre-selected category remains $C_{h}$, then one necessarily has $\sigma\left(B_{h}^{*},\{a\}\right) \geqslant \lambda, \sigma\left(B_{h-1},\{a\}\right)<\lambda$, and $\rho\left(\{a\}, B_{h}^{*}\right)>\rho\left(\{a\}, B_{h-1}\right)$, which imply that the ascending selected category remains $C_{h}$. If the descending pre-selected category becomes $C_{h}$, then one has $\sigma\left(\{a\}, B_{h}^{*}\right) \geqslant \lambda$ and $\sigma\left(\{a\}, B_{h+1}\right)<\lambda$. In such a case, if $\rho\left(\{a\}, B_{h}^{*}\right)>\rho\left(\{a\}, B_{h+1}\right)$, then the descending selected category remains $C_{h}$, which means that $\Gamma^{*}(a)=\Gamma(a)=C_{h}$ (Case IV.1); otherwise, the descend-

ing selected category becomes $C_{h+1}$. In the latter case, $\Gamma^{*}(a)$ is obtained from $\Gamma(a)$ by adding the category $C_{h+1}$, such that $\Gamma^{*}(a)=\left[C_{h}, C_{h+1}\right]($ Case IV.2).

Since the proof of the remaining cases is similar the the above ones, and taking into account that all the Cases I.-V. are true, then we achieve the proof of Theorem 3. Let us notice that the case (d) in the proof of Proposition 2 is trivially based on the cases (b) and (c) of the same proof.

\section{References}

Almeida-Dias, J., Figueira, J., Roy, B., 2010. ElEctre TrI-C: A multiple criteria sorting method based on characteristic reference actions. European Journal of Operational Research 204, 565-580.

Araz, C., Ozkarahan, I., 2007. Supplier evaluation and management system for strategic sourcing based on a new multicriteria sorting procedure. International Journal of Prodution Economics 106, 585-606.

Belacel, N., 2000. Multicriteria assignment method PROAFTN: Methodology and medical application. European Journal of Operational Research 125, $175-183$.

Błaszczyński, J., Greco, S., Słowiński, R., 2007. Multi-criteria classification: A new scheme for application of dominance-based decision rules. European Journal of Operational Research 181, 1030-1044.

Dembczyński, K., Greco, S., Słowiński, R., 2009. Rough set approach to multiple criteria classification with imprecise evaluations and assignments. European Journal of Operational Research 198, 626-636.

Doumpos, M., Zopounidis, C., 2004. A multicriteria classification approach based on pairwise comparisons. European Journal of Operational Research $158,378-389$.

Fernández, E., Navarro, J., 2011. A new approach to multi-criteria sorting based on fuzzy outranking relations: The THESEus method. European Journal of Operational Research In press. DOI: 10.1016/j.ejor.2011.03.036.

Fernández, E., Navarro, J., Bernal, S., 2009. Multicriteria sorting using a valued indifference relation under a preference disaggregation paradigm. European Journal of Operational Research 198, 602-609.

Fernández, E., Navarro, J., Duarte, A., 2008. Multicriteria sorting using a valued preference closeness relation. European Journal of Operational Research 185, 673-686.

Greco, S., Matarazzo, B., Słowiński, R., 2002. Rough sets methodology for sorting problems in presence of multiple attributes and criteria. European Journal of Operational Research 138, 247-259.

Greco, S., Mousseau, V., Słowiński, R., 2010. Multiple criteria sorting with a set of additive value functions. European Journal of Operational Research 207, 1455-1470.

Henriet, L., 2000. Systèmes d'Évaluation et de Classification Multicritères pour l'Aide à la Décision : Construction de Modèles et Procédures d'Affectation. Thèse de Doctorat. Université Paris-Dauphine. Paris, France.

Léger, J., Martel, J., 2002. A multicriteria assignment procedure for a nominal sorting problematic. European Journal of Operational Research 138, 349-364.

Nemery, P., Lamboray, C., 2008. FlowSort: A flow-based sorting method with limiting or central profiles. TOP 16, 90-113.

Perny, P., 1998. Multicriteria filtering methods based on concordance and non-discordance principles. Annals of Operations Research 80, 137-165.

Rigopoulos, G., Psarras, J., Askounis, D., 2008. Fuzzy assignment procedure based on categories boundaries. American Journal of Applied Sciences 5, $844-851$.

Roy, B., 1996. Multicriteria Methodology for Decision Aiding. Kluwer Academic Publishers, Dordrecht.

Roy, B., Bouyssou, D., 1993. Aide Multicritère à la Décision : Méthodes et Cas. Economica, Paris, France.

Yu, W., 1992. Aide Multicritère à la Décision dans le Cadre de la Problématique du Tri : Concepts, Méthodes et Applications. Thèse de Doctorat. LAMSADE, Université Paris-Dauphine. Paris, France. 